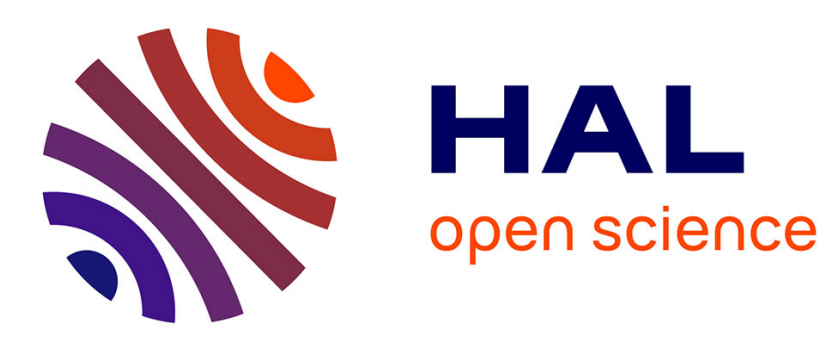

\title{
Proximal approaches for matrix optimization problems: Application to robust precision matrix estimation
} Alessandro Benfenati, Emilie Chouzenoux, Jean-Christophe Pesquet

\section{To cite this version:}

Alessandro Benfenati, Emilie Chouzenoux, Jean-Christophe Pesquet. Proximal approaches for matrix optimization problems: Application to robust precision matrix estimation. Signal Processing, 2020, 169, 10.1016/j.sigpro.2019.107417 . hal-02422403

\section{HAL Id: hal-02422403 https://hal.science/hal-02422403}

Submitted on 22 Dec 2019

HAL is a multi-disciplinary open access archive for the deposit and dissemination of scientific research documents, whether they are published or not. The documents may come from teaching and research institutions in France or abroad, or from public or private research centers.
L'archive ouverte pluridisciplinaire HAL, est destinée au dépôt et à la diffusion de documents scientifiques de niveau recherche, publiés ou non, émanant des établissements d'enseignement et de recherche français ou étrangers, des laboratoires publics ou privés. 


\title{
Proximal Approaches for Matrix Optimization Problems: Application to Robust Precision Matrix Estimation*
}

\author{
A. Benfenati ${ }^{\mathrm{a}, *}$, E. Chouzenoux ${ }^{\mathrm{b}}$, J.-C. Pesquet $^{\mathrm{b}}$ \\ ${ }^{a}$ Dipartimento di Scienze e Politiche Ambientali, Universitá degli studi di Milano, Via Celoria 2, 20133, Milano, Italy \\ ${ }^{b}$ Center for Visual Computing, INRIA Saclay and CentraleSupélec, University Paris-Saclay, 9 rue Joliot-Curie, 91190 , \\ Gif-sur-Yvette, France
}

\begin{abstract}
In recent years, there has been a growing interest in mathematical models leading to the minimization, in a symmetric matrix space, of a Bregman divergence coupled with a regularization term. We address problems of this type within a general framework where the regularization term is split into two parts, one being a spectral function while the other is arbitrary. A Douglas-Rachford approach is proposed to address such problems, and a list of proximity operators is provided allowing us to consider various choices for the fit-to-data functional and for the regularization term. Based on our theoretical results, two novel approaches are proposed for the noisy graphical lasso problem, where a covariance or precision matrix has to be statistically estimated in the presence of noise. The Douglas-Rachford approach directly applies to the estimation of the covariance matrix. When the precision matrix is sought, we solve a nonconvex optimization problem. More precisely, we propose a majorization-minimization approach building a sequence of convex surrogates and solving the inner optimization subproblems via the aforementioned Douglas-Rachford procedure. We establish conditions for the convergence of this iterative scheme. We illustrate the good numerical performance of the proposed approaches with respect to state-of-the-art approaches on synthetic and real-world datasets.
\end{abstract}

Keywords: Covariance estimation; graphical lasso; matrix optimization; Douglas-Rachford method; majorization-minimization; Bregman divergence

\section{Introduction}

In recent years, various applications such as shape classification models [1], gene expression [2], model selection [3, 4, computer vision [5], inverse covariance estimation [6, 17, 8, 9, 10, 11, 12], graph estimation

\footnotetext{
${ }^{\star}$ This work was funded by the Agence Nationale de la Recherche under grant ANR-14-CE27-0001 GRAPHSIP and it was supported by Institut Universitaire de France

${ }^{*}$ Corresponding author.

Email addresses: alessandro.benfenati@unimi.it (A. Benfenati), emilie.chouzenoux@centralesupelec.fr (E. Chouzenoux), jean-christophe@pesquet.eu (J.-C. Pesquet)
} 
[13, 14, 15, 16, social network and corporate inter-relationships analysis [17, or brain network analysis [18] have led to matrix variational formulations of the form:

$$
\underset{\mathbf{C} \in \mathcal{S}_{n}}{\operatorname{minimize}} f(\mathbf{C})-\operatorname{trace}(\mathbf{T C})+g(\mathbf{C}),
$$

where $\mathcal{S}_{n}$ is the set of real symmetric matrices of dimension $n \times n$, $\mathbf{T}$ is a given $n \times n$ real matrix (without loss of generality, it will be assumed to be symmetric), and $\left.\left.f: \mathcal{S}_{n} \rightarrow\right]-\infty,+\infty\right]$ and $g$ : $\left.\left.\mathcal{S}_{n} \rightarrow\right]-\infty,+\infty\right]$ are lower-semicontinuous functions which are proper, in the sense that they are finite at least in one point. It is worth noticing that the notion of Bregman divergence [19] gives a particular insight into Problem (1). Indeed, suppose that $f$ is a convex function differentiable on the interior of its $\operatorname{domain} \operatorname{int}(\operatorname{dom} f) \neq \varnothing$. Let us recall that, in $\mathcal{S}_{n}$ endowed with the Frobenius norm, the $f$-Bregman divergence between $\mathbf{C} \in \mathcal{S}_{n}$ and $\mathbf{Y} \in \operatorname{int}(\operatorname{dom} f)$ is

$$
D^{f}(\mathbf{C}, \mathbf{Y})=f(\mathbf{C})-f(\mathbf{Y})-\operatorname{trace}(\mathbf{T}(\mathbf{C}-\mathbf{Y})),
$$

where $\mathbf{T}=\nabla f(\mathbf{Y})$ is the gradient of $f$ at $\mathbf{Y}$. Hence, the original problem (1) is equivalently expressed as

$$
\underset{\mathbf{C} \in \mathcal{S}_{n}}{\operatorname{minimize}} g(\mathbf{C})+D^{f}(\mathbf{C}, \mathbf{Y})
$$

Solving Problem (3) amounts to computing the proximity operator of $g$ at $\mathbf{Y}$ with respect to the divergence $D^{f}$ [20, 21] in the space $\mathcal{S}_{n}$. In the vector case, such kind of proximity operator has been found to be useful in a number of recent works regarding, for example, image restoration [22, 23, 24], image reconstruction [25], and compressive sensing problems [26, 27.

In this paper, it will be assumed that $f$ belongs to the class of spectral functions [28, Chapter 5, Section 2], i.e., for every permutation matrix $\boldsymbol{\Sigma} \in \mathbb{R}^{n \times n}$,

$$
\left(\forall \mathbf{C} \in \mathcal{S}_{n}\right) \quad f(\mathbf{C})=\varphi(\boldsymbol{\Sigma} \mathbf{d}),
$$

where $\left.\left.\varphi: \mathbb{R}^{n} \rightarrow\right]-\infty,+\infty\right]$ is a proper lower semi-continuous convex function and $\mathbf{d}$ is a vector of eigenvalues of $\mathbf{C}$.

Due to the nature of the problems, in many of the aforementioned applications, $g$ is a regularization function promoting the sparsity of $\mathbf{C}$. We consider here a more generic class of regularization functions obtained by decomposing $g$ as $g_{0}+g_{1}$, where $g_{0}$ is a spectral function, i.e., for every permutation matrix $\boldsymbol{\Sigma} \in \mathbb{R}^{n \times n}$,

$$
\left(\forall \mathbf{C} \in \mathcal{S}_{n}\right) \quad g_{0}(\mathbf{C})=\psi(\boldsymbol{\Sigma} \mathbf{d}),
$$

with $\left.\left.\psi: \mathbb{R}^{n} \rightarrow\right]-\infty,+\infty\right]$ a proper lower semi-continuous function, $\mathbf{d}$ still denoting a vector of the eigenvalues of $\mathbf{C}$, while $\left.g_{1}: \mathcal{S}_{n} \rightarrow\right]-\infty,+\infty$ ] is a proper lower semi-continuous function which cannot be expressed under a spectral form.

A very popular and useful example encompassed by our framework is the graphical lasso (GLASSO) problem, where $f$ is the minus log-determinant function, $g_{1}$ is a component-wise $\ell_{1}$ norm (of the matrix 
elements), and $g_{0} \equiv 0$. Various algorithms have been proposed to solve Problem (1) in this context, including the popular GLASSO algorithm [6] and some of its recent variants [29]. We can also mention the dual block coordinate ascent method from [3], the SPICE algorithm [30, the gradient projection method in [1, the Refitted CLIME algorithm [31, various algorithms [8] based on Nesterov's smooth gradient approach [32, ADMM approaches [33, an inexact Newton method [34, and interior point methods [15, 35. A related model is addressed in 2, 4, with the additional assumption that the sought solution can be split as $\mathbf{C}_{1}+\mathbf{C}_{2}$, where $\mathbf{C}_{1}$ is sparse and $\mathbf{C}_{2}$ is low-rank. The computation of a sparse+low-rank solution is adressed in [36, 37, 38. Finally, let us mention the ADMM algorithm from [39], and the incremental proximal gradient approach from [40, both addressing Problem (1) when $f$ is the squared Frobenius norm, $g_{0}$ is a nuclear norm, and $g_{1}$ is an element-wise $\ell_{1}$ norm.

The main goal of this paper is to propose numerical approaches for solving Problem (1). Two settings will be investigated, namely $(i) g_{1} \equiv 0$, i.e. the whole cost function is a spectral one, $(i i) g_{1} \not \equiv 0$. In the former case, some general results concerning the $D^{f}$-proximity operator of $g_{0}$ are provided. In the latter case, a Douglas-Rachford (DR) optimization method is proposed, which leads us to calculate the proximity operators of several spectral functions of interest. We then consider applications of our results to the estimation of (possibly low-rank) covariance or precision matrices from noisy observations of multivariate Gaussian random variables. The novelty of our formulation lies in the fact that information on the noise is incorporated into the objective function, while preserving the desirable sparsity properties of the sought matrix. Two variational approaches are proposed for estimating either the covariance matrix or its inverse, depending on the prior assumptions made on the problem. The cost function arising from the first formulation is minimized through our proposed DR procedure under mild assumptions on the involved regularization functions. This procedure represents a valid alternative to other algorithms from the literature (see [40, 39]). In turn, the proposed objective function involved in the second formulation is proved to non-convex. Up to the best of our knowledge, no method is available in the literature to solve this problem. We thus introduce a novel majorization-minimization (MM) algorithm where the inner subproblems are solved by employing the aforementioned DR procedure, and establish convergence guarantees for this method.

The paper is organized as follows. Section 2 briefly discusses the solution of the particular instance of Problem (1) corresponding to $g_{1} \equiv 0$. Section 3 describes a proximal DR minimization algorithm allowing us to address the problem when $g_{1} \not \equiv 0$. Its implementation is discussed for a bunch of useful choices for the involved functionals. Section 4 presents two matrix minimization problems arising when estimating covariance/precision matrices from noisy realizations of a multivariate Gaussian distribution. While the first one can be solved directly with the DR approach introduced in Section 3 , the second non-convex problem is addressed thanks to a novel MM scheme, with inner steps solved with our DR method. In Section 5, a performance comparison of the DR approach for precision matrix estimation with state-ofthe-art algorithms is performed. The second part of this section is devoted to numerical experiments that 
illustrate the applicability of our MM method and its good performance on synthetic and real-world datasets.

Notation: Greek letters usually designate real numbers, bold letters designate vectors in a Euclidean space, capital bold letters indicate matrices. The $i$-th element of the vector $\mathbf{d}$ is denoted by $d_{i}$. $\operatorname{Diag}(\mathbf{d})$ denotes the diagonal matrix whose diagonal elements are the components of $\mathbf{d}$. $\mathcal{D}_{n}$ is the cone of vectors $\mathbf{d} \in \mathbb{R}^{n}$ whose components are ordered by decreasing values. The symbol vect $(\mathbf{C})$ denotes the vector resulting from a column-wise ordering of the elements of matrix $\mathbf{C}$. The product $\mathbf{A} \otimes \mathbf{B}$ denotes the classical Kronecker product of matrices $\mathbf{A}$ and $\mathbf{B}$, while $\mathbf{A} \odot \mathbf{B}$ denotes the Hadamard component-wise product. Let $\mathcal{H}$ be a real Hilbert space endowed with an inner product $\langle\cdot, \cdot\rangle$ and a norm $\|\cdot\|$, the domain of a function $f: \mathcal{H} \rightarrow]-\infty,+\infty]$ is $\operatorname{dom} f=\{x \in \mathcal{H} \mid f(x)<+\infty\} . f$ is coercive if $\lim _{\|x\| \rightarrow+\infty} f(x)=+\infty$ and supercoercive if $\lim _{\|x\| \rightarrow+\infty} f(x) /\|x\|=+\infty$. The Moreau subdifferential of $f$ at $x \in \mathcal{H}$ is $\partial f(x)=\{t \in \mathcal{H} \mid(\forall y \in \mathcal{H}) f(y) \geq$ $f(x)+\langle t, y-x\rangle\} . \Gamma_{0}(\mathcal{H})$ denotes the class of lower-semicontinuous convex functions from $\mathcal{H}$ to $\left.]-\infty,+\infty\right]$ with a nonempty domain (proper). If $f \in \Gamma_{0}(\mathcal{H})$ is (Gâteaux) differentiable at $x \in \mathcal{H}$, then $\partial f(x)=\{\nabla f(x)\}$ where $\nabla f(x)$ is the gradient of $f$ at $x$. If a function $f: \mathcal{H} \rightarrow]-\infty,+\infty]$ possesses a unique minimizer on a set $E \subset \mathcal{H}$, it will be denoted by $\operatorname{argmin} f(x)$. If there are possibly several minimizers, their set will be denoted by $\operatorname{Argmin} f(x)$. Given a set $E, \operatorname{int}(E)$ designates the interior of $E$ and $\iota_{E}$ denotes the indicator function of the set, which is equal to 0 over this set and $+\infty$ otherwise. In the remainder of the paper, the underlying Hilbert space will be $\mathcal{S}_{n}$, the set of real symmetric matrices equipped with the Frobenius norm, denoted by $\|\cdot\|_{\mathrm{F}}$. The matrix spectral norm is denoted by $\|\cdot\|_{\mathrm{S}}$, the $\ell_{1}$ norm of a matrix $\mathbf{A}=\left(A_{i, j}\right)_{i, j}$ is $\|\mathbf{A}\|_{1}=\sum_{i, j}\left|A_{i, j}\right|$. For every $p \in\left[1,+\infty\left[, \mathcal{R}_{p}(\mathbf{A})\right.\right.$ denotes the Schatten $p$-norm of $\mathbf{A}$, the nuclear norm being obtained when $p=1$. $\mathcal{O}_{n}$ denotes the set of orthogonal matrices of dimension $n$ with real elements; $\mathcal{S}_{n}^{+}$ and $\mathcal{S}_{n}^{++}$denote the set of real symmetric positive semidefinite, and symmetric positive definite matrices, respectively, of dimension $n$. $\mathrm{I}_{\mathrm{d}}$ denotes the identity matrix whose dimension will be understood from the context. The soft thresholding operator soft $\mu$ and the hard thresholding operator hard ${ }_{\mu}$ of parameter $\mu \in\left[0,+\infty\left[\right.\right.$ are given by $(\forall \xi \in \mathbb{R}) \operatorname{soft}_{\mu}(\xi)=\operatorname{sign}(\xi) \max \{|\xi|-\mu, 0\}$ and $\operatorname{hard}_{\mu}(\xi)=\xi_{\iota}\{\xi \mid>\mu\}$, respectively.

\section{Spectral Approach}

In this section, we show that, in the particular case when $g_{1} \equiv 0$, Problem (1) reduces to the optimization of a function defined on $\mathbb{R}^{n}$. Indeed, the problem then reads:

$$
\underset{\mathbf{C} \in \mathcal{S}_{n}}{\operatorname{minimize}} f(\mathbf{C})-\operatorname{trace}(\mathbf{T C})+g_{0}(\mathbf{C}),
$$

where the spectral forms of $f$ and $g_{0}$ allow us to take advantage of the eigendecompositions of $\mathbf{C}$ and $\mathbf{T}$ in order to simplify the optimization problem, as stated below. Since the results in this section are direct extension of existing ones, the proofs will be skipped. The reader can find more details in the extended version of the paper 41 . 
Theorem 1. Let $\mathbf{t} \in \mathbb{R}^{n}$ be a vector of eigenvalues of $\mathbf{T}$ and let $\mathbf{U}_{\mathbf{T}} \in \mathcal{O}_{n}$ be such that $\mathbf{T}=\mathbf{U}_{T} \operatorname{Diag}(\mathbf{t}) \mathbf{U}_{T}^{\top}$. Let $f$ and $g_{0}$ be functions satisfying (4) and (5), respectively, where $\varphi$ and $\psi$ are lower-semicontinuous functions. Assume that $\operatorname{dom} \varphi \cap \operatorname{dom} \psi \neq \varnothing$ and that the function $\mathbf{d} \mapsto \varphi(\mathbf{d})-\mathbf{d}^{\top} \mathbf{t}+\psi(\mathbf{d})$ is coercive. Then a solution to Problem (6) exists, which is given by

$$
\widehat{\mathbf{C}}=\mathbf{U}_{\mathbf{T}} \operatorname{Diag}(\widehat{\mathbf{d}}) \mathbf{U}_{\mathbf{T}}^{\top}
$$

where $\widehat{\mathbf{d}}$ is any solution to the following problem:

$$
\underset{\mathbf{d} \in \mathbb{R}^{n}}{\operatorname{minimize}} \varphi(\mathbf{d})-\mathbf{d}^{\top} \mathbf{t}+\psi(\mathbf{d})
$$

Before deriving a main consequence of Theorem[1, we need to recall some definitions from convex analysis [42, Chapter 26] [20, Section 3.4]:

Definition 1. Let $\mathcal{H}$ be a finite dimensional real Hilbert space with norm $\|\cdot\|$ and scalar product $\langle\cdot, \cdot\rangle$. Let $h: \mathcal{H} \rightarrow]-\infty,+\infty]$ be a proper convex function.

- $h$ is essentially smooth if $h$ is differentiable on $\operatorname{int}(\operatorname{dom} h) \neq \varnothing \quad$ and $\lim _{n \rightarrow+\infty}\left\|\nabla h\left(x_{n}\right)\right\|=+\infty$ for every sequence $\left(x_{n}\right)_{n \in \mathbb{N}}$ of $\operatorname{int}(\operatorname{dom} h)$ converging to a point on the boundary of dom $h$.

- $h$ is essentially strictly convex if $h$ is strictly convex on every convex subset of the domain of its subdifferential.

- $h$ is a Legendre function if it is both essentially smooth and essentially strictly convex.

- If $h$ is differentiable on $\operatorname{int}(\operatorname{dom} h) \neq \varnothing$, the $h$-Bregman divergence is the function $D^{h}$ defined on $\mathcal{H}^{2}$ as

$$
\left(\forall(x, y) \in \mathcal{H}^{2}\right)
$$

$$
D^{h}(x, y)= \begin{cases}h(x)-h(y)-\langle\nabla h(y), x-y\rangle & \text { if } y \in \operatorname{int}(\operatorname{dom} f) \\ +\infty & \text { otherwise }\end{cases}
$$

- Assume that $h$ is a lower-semicontinuous convex Legendre function and that $\ell$ is a lower-semicontinuous convex function such that $\operatorname{int}(\operatorname{dom} h) \cap \operatorname{dom} \ell \neq \varnothing$ and either $\ell$ is bounded from below or $h+\ell$ is supercoercive. Then, the $D^{h}$-proximity operator of $\ell$ is

$$
\begin{aligned}
\operatorname{prox}_{\ell}^{h}: \operatorname{int}(\operatorname{dom} h) & \rightarrow \operatorname{int}(\operatorname{dom} h) \cap \operatorname{dom} \ell \\
y & \mapsto \underset{x \in \mathcal{H}}{\operatorname{argmin}} \ell(x)+D^{h}(x, y) .
\end{aligned}
$$


In this definition, when $h=\|\cdot\|^{2} / 2$, we recover the classical definition of the proximity operator in [43, which is defined over $\mathcal{H}$, for every function $\ell \in \Gamma_{0}(\mathcal{H})$, and that will be simply denoted by prox $_{\ell}$.

As an offspring of Theorem 1, we then get:

Corollary 1. Let $f$ and $g_{0}$ be functions satisfying (4) and (5), respectively, where $\varphi \in \Gamma_{0}\left(\mathbb{R}^{n}\right)$ is a Legendre function, $\psi \in \Gamma_{0}\left(\mathbb{R}^{n}\right)$, $\operatorname{int}(\operatorname{dom} \varphi) \cap \operatorname{dom} \psi \neq \varnothing$, and either $\psi$ is bounded from below or $\varphi+\psi$ is supercoercive. Then, the $D^{f}$-proximity operator of $g_{0}$ is defined at every $\mathbf{Y} \in \mathcal{S}_{n}$ such that $\mathbf{Y}=\mathbf{U}_{\mathbf{Y}} \operatorname{Diag}(\mathbf{y}) \mathbf{U}_{\mathbf{Y}}^{\top}$ with $\mathbf{U}_{\mathbf{Y}} \in \mathcal{O}_{n}$ and $\mathbf{y} \in \operatorname{int}(\operatorname{dom} \varphi)$, and it is expressed as

$$
\operatorname{prox}_{g_{0}}^{f}(\mathbf{Y})=\mathbf{U}_{\mathbf{Y}} \operatorname{Diag}\left(\operatorname{prox}_{\psi}^{\varphi}(\mathbf{y})\right) \mathbf{U}_{\mathbf{Y}}^{\top}
$$

Remark 1. Corollary 1 extends known results concerning the case when $f=$ $\|\cdot\|_{\mathrm{F}} / 2$ [44]. A rigorous derivation of the proximity operator of spectral functions in $\Gamma_{0}\left(\mathcal{S}_{n}\right)$ for the standard Frobenius metric can be found in 455, Corollary 24.65]. We recover a similar result by adopting a more general approach. In particular, it is worth noticing that Theorem 1 does not require any convexity assumption.

\section{Proximal Iterative Approach}

Let us now turn our attention to the more general case of the resolution of Problem (1) when $f \in \Gamma_{0}\left(\mathcal{S}_{n}\right)$ and $g_{1} \not \equiv 0$. Proximal splitting approaches for finding a minimizer of a sum of non-necessarily smooth functions have attracted a large interest in the last years [46, 47, 48, 49]. In these methods, the functions can be dealt with either via their gradient or their proximity operator depending on their differentiability properties. In this section, we first list a number of proximity operators of scaled versions of $f$-trace $(\mathbf{T} \cdot)+g_{0}$, where $f$ and $g_{0}$, satisfying (4) and (5), are chosen among several options that can be useful in a wide range of practical scenarios. Based on these results, we then propose a proximal splitting Douglas-Rachford algorithm to solve Problem (1).

\subsection{Proximity Operators}

By definition, computing the proximity operator of $\gamma\left(f-\operatorname{trace}(\mathbf{T} \cdot)+g_{0}\right)$ with $\left.\gamma \in\right] 0,+\infty\left[\right.$ at $\overline{\mathbf{C}} \in \mathcal{S}_{n}$ amounts to find a minimizer of the function

$$
\mathbf{C} \mapsto f(\mathbf{C})-\operatorname{trace}(\mathbf{T C})+g_{0}(\mathbf{C})+\frac{1}{2 \gamma}\|\mathbf{C}-\overline{\mathbf{C}}\|_{\mathrm{F}}^{2}
$$

over $\mathcal{S}_{n}$. The (possibly empty) set of such minimizers is denoted by $\operatorname{Prox}_{\gamma\left(f-\operatorname{trace}(\mathbf{T} \cdot)+g_{0}\right)}(\overline{\mathbf{C}})$. As pointed out in Section 2 if $f+g_{0} \in \Gamma_{0}\left(\mathcal{S}_{n}\right)$ then this set is the singleton $\left\{\operatorname{prox}_{\gamma\left(f-\operatorname{trace}(\mathbf{T} \cdot)+g_{0}\right)}(\overline{\mathbf{C}})\right\}$. We have the following characterization of this proximity operator: 
Theorem 2. Let $\gamma \in] 0,+\infty\left[\right.$ and $\overline{\mathbf{C}} \in \mathcal{S}_{n}$. Let $f$ and $g_{0}$ be functions satisfying (4) and (5), respectively, where $\varphi \in \Gamma_{0}\left(\mathbb{R}^{n}\right)$ and $\psi$ is a lower-semicontinuous function such that $\operatorname{dom} \varphi \cap \operatorname{dom} \psi \neq \varnothing$. Let $\boldsymbol{\lambda} \in \mathbb{R}^{n}$ and $\mathbf{U} \in \mathcal{O}_{n}$ be such that $\overline{\mathbf{C}}+\gamma \mathbf{T}=\mathbf{U} \operatorname{Diag}(\boldsymbol{\lambda}) \mathbf{U}^{\top}$.

(i) If $\psi$ is lower bounded by an affine function then $\operatorname{Prox}_{\gamma(\varphi+\psi)}(\boldsymbol{\lambda}) \neq \varnothing$ and, for every $\widehat{\boldsymbol{\lambda}} \in \operatorname{Prox}_{\gamma(\varphi+\psi)}(\boldsymbol{\lambda})$,

$$
\mathbf{U} \operatorname{Diag}(\widehat{\boldsymbol{\lambda}}) \mathbf{U}^{\top} \in \operatorname{Prox}_{\gamma\left(f-\operatorname{trace}(\mathbf{T} \cdot)+g_{0}\right)}(\overline{\mathbf{C}}) .
$$

(ii) If $\psi$ is convex, then

$$
\operatorname{prox}_{\gamma\left(f-\operatorname{trace}(\mathbf{T} \cdot)+g_{0}\right)}(\overline{\mathbf{C}})=\mathbf{U} \operatorname{Diag}\left(\operatorname{prox}_{\gamma(\varphi+\psi)}(\boldsymbol{\lambda})\right) \mathbf{U}^{\top} .
$$

Proof. See Appendix A.

We will next focus on the use of Theorem 2 for three choices for $f$, namely the classical squared Frobenius norm, the minus log det functional, and the Von Neumann entropy, each choice being coupled with various possible choices for $g_{0}$.

\subsubsection{First Example: Squared Frobenius Norm}

A suitable choice in Problem (1) is $f=\|\cdot\|_{\mathrm{F}}^{2} / 2$ [39, 40, 50. The squared Froebenius norm is the spectral function associated with the function $\varphi=\|\cdot\|^{2} / 2$. It is worth mentioning that this choice for $f$ allows us to rewrite the original Problem (1) under the form (3), where

$$
\left(\forall(\mathbf{C}, \mathbf{Y}) \in \mathcal{S}_{n}^{2}\right) \quad D^{f}(\mathbf{C}, \mathbf{Y})=\frac{1}{2}\|\mathbf{C}-\mathbf{Y}\|_{\mathrm{F}}^{2} .
$$

We have thus re-expressed Problem (1) as the determination of a proximal point of function $g$ at $\mathbf{T}$ in the Frobenius metric. Table 1 presents several examples of spectral functions $g_{0}$ and the expression of the proximity operator of $\gamma(\varphi+\psi)$ with $\gamma \in] 0,+\infty$ [. These expressions were established by using the properties of proximity operators of functions defined on $\mathbb{R}^{n}$ (see [51, Example 4.4] and [46, Tables 10.1 and 10.2]).

Remark 2. Another option for $g_{0}$ is to choose it equal to $\mu\|\cdot\|_{\mathrm{S}}$ where $\left.\mu \in\right] 0,+\infty[$. For every $\gamma \in] 0,+\infty[$, we have then

$$
\left(\forall \boldsymbol{\lambda} \in \mathbb{R}^{n}\right) \quad \operatorname{prox}_{\gamma(\varphi+\psi)}(\boldsymbol{\lambda})=\operatorname{prox}_{\frac{\mu \gamma}{1+\gamma}\|\cdot\|_{+\infty}}\left(\frac{\boldsymbol{\lambda}}{1+\gamma}\right),
$$

where $\|\cdot\|_{+\infty}$ is the infinity norm of $\mathbb{R}^{n}$. By noticing that $\|\cdot\|_{+\infty}$ is the conjugate function of the indicator function of $B_{\ell^{1}}$, the unit $\ell^{1}$ ball centered at 0 of $\mathbb{R}^{n}$, and using Moreau's decomposition formula, 45. Proposition 24.8(ix)] yields

$$
\left(\forall \boldsymbol{\lambda} \in \mathbb{R}^{n}\right) \quad \operatorname{prox}_{\gamma(\varphi+\psi)}(\boldsymbol{\lambda})=\frac{1}{1+\gamma}\left(\boldsymbol{\lambda}-\mu \gamma \operatorname{proj}_{B_{\ell^{1}}}\left(\frac{\boldsymbol{\lambda}}{\mu \gamma}\right)\right) .
$$

The required projection onto $B_{\ell^{1}}$ can be computed through efficient algorithms [52, 53]. 
Table 1: Proximity operators of $\gamma\left(\frac{1}{2}\|\cdot\|_{\mathrm{F}}^{2}+g_{0}\right)$ with $\gamma>0$ evaluated at symmetric matrix with vector of eigenvalues $\boldsymbol{\lambda}=\left(\lambda_{i}\right)_{1 \leq i \leq n}$. For the inverse Schatten penalty, the function is set to $+\infty$ when the argument $\mathbf{C}$ is not positive definite. $E_{1}$ denotes the set of matrices in $\mathcal{S}_{n}$ with Frobenius norm less than or equal to $\alpha$ and $E_{2}$ the set of matrices in $\mathcal{S}_{n}$ with eigenvalues between $\alpha$ and $\beta$. In the last line, the $i$-th component of the proximity operator is obtained by searching among the nonnegative roots of a third order polynomial those minimizing $\lambda_{i}^{\prime} \mapsto \frac{1}{2}\left(\lambda_{i}^{\prime}-\left|\lambda_{i}\right|\right)^{2}+\gamma\left(\frac{1}{2}\left(\lambda_{i}^{\prime}\right)^{2}+\mu \log \left(\left(\lambda_{i}^{\prime}\right)^{2}+\varepsilon\right)\right)$.

\begin{tabular}{|c|c|}
\hline$g_{0}(\mathbf{C}), \mu>0$ & $\operatorname{prox}_{\gamma(\varphi+\psi)}(\boldsymbol{\lambda})$ \\
\hline $\begin{array}{l}\text { Nuclear norm } \\
\quad \mu \mathcal{R}_{1}(\mathbf{C})\end{array}$ & $\left(\operatorname{soft}_{\frac{\mu \gamma}{\gamma+1}}\left(\frac{\lambda_{i}}{\gamma+1}\right)\right)_{1 \leq i \leq n}$ \\
\hline $\begin{array}{l}\text { Frobenius norm } \\
\qquad \mu\|\mathbf{C}\|_{\mathrm{F}}\end{array}$ & $\left(1-\frac{\gamma \mu}{\|\boldsymbol{\lambda}\|}\right) \frac{\boldsymbol{\lambda}}{1+\gamma}$ if $\|\boldsymbol{\lambda}\|>\gamma \mu$ and $\mathbf{0}$ otherwise \\
\hline $\begin{array}{l}\text { Squared Frobenius norm } \\
\qquad \mu\|\mathbf{C}\|_{\mathrm{F}}^{2} \\
\end{array}$ & $\frac{\boldsymbol{\lambda}}{1+\gamma(1+2 \mu)}$ \\
\hline $\begin{array}{c}\text { Schatten 3-penalty } \\
\mu \mathcal{R}_{3}^{3}(\mathbf{C}) \\
\end{array}$ & $(6 \gamma \mu)^{-1}\left(\operatorname{sign}\left(\lambda_{i}\right) \sqrt{(\gamma+1)^{2}+12\left|\lambda_{i}\right| \gamma \mu}-\gamma-1\right)_{1 \leq i \leq n}$ \\
\hline $\begin{array}{l}\text { Schatten } 4 \text {-penalty } \\
\qquad \mu \mathcal{R}_{4}^{4}(\mathbf{C})\end{array}$ & $(8 \gamma \mu)^{-1 / 3}\left(\sqrt[3]{\lambda_{i}+\sqrt{\lambda_{i}^{2}+\zeta}}+\sqrt[3]{\lambda_{i}-\sqrt{\lambda_{i}^{2}+\zeta}}\right)_{1 \leq i \leq n}$ with $\zeta=\frac{(\gamma+1)^{3}}{27 \gamma \mu}$ \\
\hline $\begin{array}{l}\text { Schatten } 4 / 3-\text { penalty } \\
\qquad \mu \mathcal{R}_{4 / 3}^{4 / 3}(\mathbf{C}) \\
\end{array}$ & $\begin{array}{c}\frac{1}{1+\gamma}\left(\lambda_{i}+\frac{4 \gamma \mu}{3 \sqrt[3]{2(1+\gamma)}}\left(\sqrt[3]{\sqrt{\lambda_{i}^{2}+\zeta}-\lambda_{i}}-\sqrt[3]{\sqrt{\lambda_{i}^{2}+\zeta}+\lambda_{i}}\right)\right)_{1 \leq i \leq n} \\
\text { with } \zeta=\frac{256(\gamma \mu)^{3}}{729(1+\gamma)}\end{array}$ \\
\hline $\begin{array}{l}\text { Schatten } 3 / 2-\text { penalty } \\
\qquad \mu \mathcal{R}_{3 / 2}^{3 / 2}(\mathbf{C})\end{array}$ & $\frac{1}{1+\gamma}\left(\lambda_{i}+\frac{9 \gamma^{2} \mu^{2}}{8(1+\gamma)} \operatorname{sign}\left(\lambda_{i}\right)\left(1-\sqrt{1+\frac{16(1+\gamma)}{9 \gamma^{2} \mu^{2}}\left|\lambda_{i}\right|}\right)\right)_{1 \leq i \leq n}$ \\
\hline $\begin{array}{c}\text { Schatten } p \text {-penalty } \\
\mu \mathcal{R}_{p}^{p}(\mathbf{C}), p \geq 1 \\
\end{array}$ & $\begin{array}{c}\left(\operatorname{sign}\left(\lambda_{i}\right) d_{i}\right)_{1 \leq i \leq n} \\
\text { with }(\forall i \in\{1, \ldots, n\}) d_{i} \geq 0 \text { and } \mu \gamma p d_{i}^{p-1}+(\gamma+1) d_{i}=\lambda_{i}\end{array}$ \\
\hline $\begin{array}{l}\text { Inverse Schatten } p \text {-penalty } \\
\qquad \mu \mathcal{R}_{p}^{p}\left(\mathbf{C}^{-1}\right), p>0\end{array}$ & $\begin{array}{c}\left(d_{i}\right)_{1 \leq i \leq n} \\
\text { with }(\forall i \in\{1, \ldots, n\}) d_{i}>0 \text { and }(\gamma+1) d_{i}^{p+2}-\lambda_{i} d_{i}^{p+1}=\mu \gamma p\end{array}$ \\
\hline $\begin{array}{l}\text { Bound on the Frobenius norm } \\
\qquad \iota_{E_{1}}(\mathbf{C})\end{array}$ & $\alpha \frac{\boldsymbol{\lambda}}{\|\boldsymbol{\lambda}\|}$ if $\|\boldsymbol{\lambda}\|>\alpha(1+\gamma)$ and $\frac{\boldsymbol{\lambda}}{1+\gamma}$ otherwise, $\alpha \in[0,+\infty[$ \\
\hline $\begin{array}{l}\text { Bounds on eigenvalues } \\
\qquad \iota_{E_{2}}(\mathbf{C})\end{array}$ & $\left(\min \left(\max \left(\lambda_{i} /(\gamma+1), \alpha\right), \beta\right)\right)_{1 \leq i \leq n},[\alpha, \beta] \subset[-\infty,+\infty]$ \\
\hline $\begin{array}{c}\operatorname{Rank} \\
\mu \operatorname{rank}(\mathbf{C}) \\
\end{array}$ & $\left(\operatorname{hard}_{\sqrt{\frac{2 \mu \gamma}{1+\gamma}}}\left(\frac{\lambda_{i}}{1+\gamma}\right)\right)_{1 \leq i \leq n}$ \\
\hline $\begin{array}{c}\text { Cauchy } \\
\mu \log \operatorname{det}\left(\mathbf{C}^{2}+\varepsilon \mathrm{I}_{\mathrm{d}}\right), \varepsilon>0\end{array}$ & $\begin{aligned} \in\{\{ & \left(\operatorname{sign}\left(\lambda_{i}\right) d_{i}\right)_{1 \leq i \leq n} \mid(\forall i \in\{1, \ldots, n\}) d_{i} \geq 0 \text { and } \\
& \left.(\gamma+1) d_{i}^{3}-\left|\lambda_{i}\right| d_{i}^{2}+(2 \gamma \mu+\varepsilon(\gamma+1)) d_{i}=\left|\lambda_{i}\right| \varepsilon\right\}\end{aligned}$ \\
\hline
\end{tabular}




\subsubsection{Second Example: Logdet Function}

Another popular choice for $f$ is the negative logarithmic determinant function [1, 33, 2, 13, 3, 6, 15, 4, which is defined as follows

$$
\left(\forall \mathbf{C} \in \mathcal{S}_{n}\right) \quad f(\mathbf{C})= \begin{cases}-\log \operatorname{det}(\mathbf{C}) & \text { if } \mathbf{C} \in \mathcal{S}_{n}^{++} \\ +\infty & \text { otherwise }\end{cases}
$$

The above function satisfies property (5) with

$$
\left(\forall \boldsymbol{\lambda}=\left(\lambda_{i}\right)_{1 \leq i \leq n} \in \mathbb{R}^{n}\right) \quad \varphi(\boldsymbol{\lambda})= \begin{cases}-\sum_{i=1}^{n} \log \left(\lambda_{i}\right) & \text { if } \boldsymbol{\lambda} \in] 0,+\infty\left[^{n}\right. \\ +\infty & \text { otherwise. }\end{cases}
$$

Actually, for a given positive definite matrix, the value of function (18) simply reduces to the Burg entropy of its eigenvalues. Here again, if $\mathbf{Y} \in \mathcal{S}_{n}^{++}$and $\mathbf{T}=-\mathbf{Y}^{-1}$, we can rewrite Problem (1) under the form (3), so that it becomes equivalent to the computation of the proximity operator of $g$ with respect to the Bregman divergence given by

$$
\left(\forall \mathbf{C} \in \mathcal{S}_{n}\right) \quad D^{f}(\mathbf{C}, \mathbf{Y})= \begin{cases}\log \left(\frac{\operatorname{det}(\mathbf{Y})}{\operatorname{det}(\mathbf{C})}\right)+\operatorname{trace}\left(\mathbf{Y}^{-1} \mathbf{C}\right)-n & \text { if } \mathbf{C} \in \mathcal{S}_{n}^{++} \\ +\infty & \text { otherwise. }\end{cases}
$$

In Table 2, we list some particular choices for $g_{0}$, and provide the associated closed form expression of the proximity operator $\operatorname{prox}_{\gamma(\varphi+\psi)}$ for $\left.\gamma \in\right] 0,+\infty[$, where $\varphi$ is defined in $(19)$. These expressions were derived from [46, Table 10.2].

Remark 3. Let $g_{0}$ be any of the convex spectral functions listed in Table 2. Let $\mathbf{W}$ be an invertible matrix in $\mathbb{R}^{n \times n}$, and let $\overline{\mathbf{C}} \in \mathcal{S}_{n}$ From the above results, one can deduce the minimizer of $\mathbf{C} \mapsto \gamma\left(f(\mathbf{C})+g_{0}\left(\mathbf{W C W} \mathbf{W}^{\top}\right)\right)+$ $\frac{1}{2}\left\|\mathbf{W C W}^{\top}-\overline{\mathbf{C}}\right\|_{\mathrm{F}}^{2}$ where $\left.\gamma \in\right] 0,+\infty[$. Indeed, by making a change of variable and by using basic properties of the $\log \operatorname{det}$ function, this minimizer is equal to $\mathbf{W}^{-1} \operatorname{prox}_{\gamma\left(f+g_{0}\right)}(\overline{\mathbf{C}})\left(\mathbf{W}^{-1}\right)^{\top}$.

\subsubsection{Third Example: Von Neumann Entropy}

Our third example is the negative Von Neumann entropy, which appears to be useful in some quantum mechanics problems [54]. It is defined as

$$
\left(\forall \mathbf{C} \in \mathcal{S}_{n}\right) \quad f(\mathbf{C})= \begin{cases}\operatorname{trace}(\mathbf{C} \log (\mathbf{C})) & \text { if } \mathbf{C} \in \mathcal{S}_{n}^{+} \\ +\infty & \text { otherwise. }\end{cases}
$$

In the above expression, if $\mathbf{C}=\mathbf{U} \operatorname{Diag}(\boldsymbol{\lambda}) \mathbf{U}^{\top}$ with $\left.\boldsymbol{\lambda}=\left(\lambda_{i}\right)_{1 \leq i \leq n} \in\right] 0,+\infty\left[^{n}\right.$ and $\mathbf{U} \in \mathcal{O}_{n}$, then $\log (\mathbf{C})=$ $\mathbf{U} \operatorname{Diag}\left(\left(\log \lambda_{i}\right)_{1 \leq i \leq n}\right) \mathbf{U}^{\top}$. The logarithm of a symmetric definite positive matrix is uniquely defined and 
Table 2: Proximity operators of $\gamma\left(f+g_{0}\right)$ with $\gamma>0$ and $f$ given by 18 , evaluated at a symmetric matrix with vector of eigenvalues $\boldsymbol{\lambda}=\left(\lambda_{i}\right)_{1 \leq i \leq n}$. For the inverse Schatten penalty, the function is set to $+\infty$ when the argument $\mathbf{C}$ is not positive definite. $E_{2}$ denotes the set of matrices in $\mathcal{S}_{n}$ with eigenvalues between $\alpha$ and $\beta$. In the last line, the $i$-th component of the proximity operator is obtained by searching among the positive roots of a fourth order polynomial those minimizing $\lambda_{i}^{\prime} \mapsto \frac{1}{2}\left(\lambda_{i}^{\prime}-\lambda_{i}\right)^{2}+\gamma\left(\mu \log \left(\left(\lambda_{i}^{\prime}\right)^{2}+\varepsilon\right)-\log \lambda_{i}^{\prime}\right)$.

\begin{tabular}{|c|c|}
\hline$g_{0}(\mathbf{C}), \mu>0$ & $\operatorname{prox}_{\gamma(\varphi+\psi)}(\boldsymbol{\lambda})$ \\
\hline $\begin{array}{l}\text { Nuclear norm } \\
\quad \mu \mathcal{R}_{1}(\mathbf{C})\end{array}$ & $\frac{1}{2}\left(\lambda_{i}-\gamma \mu+\sqrt{\left(\lambda_{i}-\gamma \mu\right)^{2}+4 \gamma}\right)_{1 \leq i \leq n}$ \\
\hline $\begin{array}{l}\text { Squared Frobenius norm } \\
\qquad \mu\|\mathbf{C}\|_{\mathrm{F}}^{2} \\
\end{array}$ & $\frac{1}{2(2 \gamma \mu+1)}\left(\lambda_{i}+\sqrt{\lambda_{i}^{2}+4 \gamma(2 \gamma \mu+1)}\right)_{1 \leq i \leq n}$ \\
\hline $\begin{array}{l}\text { Schatten } p \text {-penalty } \\
\qquad \mu \mathcal{R}_{p}^{p}(\mathbf{C}), p \geq 1\end{array}$ & $\begin{array}{c}\left(d_{i}\right)_{1 \leq i \leq n} \\
\text { with }(\forall i \in\{1, \ldots, n\}) d_{i}>0 \text { and } \mu \gamma p d_{i}^{p}+d_{i}^{2}-\lambda_{i} d_{i}=\gamma\end{array}$ \\
\hline $\begin{array}{l}\text { Inverse Schatten } p \text {-penalty } \\
\qquad \mu \mathcal{R}_{p}^{p}\left(\mathbf{C}^{-1}\right), p>0\end{array}$ & $\begin{array}{c}\left(d_{i}\right)_{1 \leq i \leq n} \\
\text { with }(\forall i \in\{1, \ldots, n\}) d_{i}>0 \text { and } d_{i}^{p+2}-\lambda_{i} d_{i}^{p+1}-\gamma d_{i}^{p}=\mu \gamma p\end{array}$ \\
\hline $\begin{array}{c}\text { Bounds on eigenvalues } \\
\iota_{E_{2}}(\mathbf{C}) \\
\end{array}$ & $\left(\min \left(\max \left(\frac{1}{2}\left(\lambda_{i}+\sqrt{\lambda_{i}^{2}+4 \gamma}\right), \alpha\right), \beta\right)\right)_{1 \leq i \leq n},[\alpha, \beta] \subset[0,+\infty]$ \\
\hline $\begin{array}{c}\text { Cauchy } \\
\mu \log \operatorname{det}\left(\mathbf{C}^{2}+\varepsilon \mathrm{I}_{\mathrm{d}}\right), \varepsilon>0\end{array}$ & $\begin{array}{l}\in\left\{\left(d_{i}\right)_{1 \leq i \leq n} \mid(\forall i \in\{1, \ldots, n\}) d_{i}>0 \text { and }\right. \\
\left.\quad d_{i}^{4}-\lambda d_{i}^{3}+(\varepsilon+\gamma(2 \mu-1)) d_{i}^{2}-\varepsilon \lambda_{i} d_{i}=\gamma \varepsilon\right\}\end{array}$ \\
\hline
\end{tabular}

the function $\mathbf{C} \mapsto \mathbf{C} \log (\mathbf{C})$ can be extended by continuity on $\mathcal{S}_{n}^{+}$similarly to the case when $n=1$. Thus, $f$ is the spectral function associated with

$$
\left(\forall \boldsymbol{\lambda}=\left(\lambda_{i}\right)_{1 \leq i \leq n} \in \mathbb{R}^{n}\right) \quad \varphi(\boldsymbol{\lambda})= \begin{cases}\sum_{i=1}^{n} \lambda_{i} \log \left(\lambda_{i}\right) & \text { if } \boldsymbol{\lambda} \in\left[0,+\infty\left[^{n}\right.\right. \\ +\infty & \text { otherwise }\end{cases}
$$

Note that the Von Neumann entropy defined for symmetric matrices is simply equal to the well-known Shannon entropy [55] of the input eigenvalues. With this choice for function $f$, by setting $\mathbf{T}=\log (\mathbf{Y})+\mathrm{I}_{\mathrm{d}}$ where $\mathbf{Y} \in \mathcal{S}_{n}^{++}$, Problem (1) can be recast under the form (3), so that it becomes equivalent to the computation of the proximity operator of $g$ with respect to the Bregman divergence associated with the Von Neumann entropy:

$\left(\forall \mathbf{C} \in \mathcal{S}_{n}\right) \quad D^{f}(\mathbf{C}, \mathbf{Y})=$

$$
\begin{cases}\operatorname{trace}\left(\mathbf{C} \log (\mathbf{C})-\mathbf{Y} \log (\mathbf{Y})-\left(\log (\mathbf{Y})+\mathrm{I}_{\mathrm{d}}\right)(\mathbf{C}-\mathbf{Y})\right) & \text { if } \mathbf{C} \in \mathcal{S}_{n}^{+} \\ +\infty & \text { otherwise. }\end{cases}
$$

We provide in Table 3 a list of closed form expressions of the proximity operator of $\gamma\left(f+g_{0}\right)$ for several choices of the spectral function $g_{0}$. 
Table 3: Proximity operators of $\gamma\left(f+g_{0}\right)$ with $\gamma>0$ and $f$ given by 21, evaluated at a symmetric matrix with vector of eigenvalues $\boldsymbol{\lambda}=\left(\lambda_{i}\right)_{1 \leq i \leq n}$. $E_{2}$ denotes the set of matrices in $\mathcal{S}_{n}$ with eigenvalues between $\alpha$ and $\beta$. W $(\cdot)$ denotes the W-Lambert function [56].

\begin{tabular}{|c|c|}
\hline$g_{0}(\mathbf{C}), \mu>0$ & $\operatorname{prox}_{\gamma(\varphi+\psi)}(\boldsymbol{\lambda})$ \\
\hline $\begin{array}{l}\text { Nuclear norm } \\
\qquad \mu \mathcal{R}_{1}(\mathbf{C})\end{array}$ & $\gamma\left(\mathrm{W}\left(\frac{1}{\gamma} \exp \left(\frac{\lambda_{i}}{\gamma}-\mu-1\right)\right)\right)_{1 \leq i \leq n}$ \\
\hline $\begin{array}{l}\text { Squared Frobenius norm } \\
\qquad \mu\|\mathbf{C}\|_{\mathrm{F}}^{2}\end{array}$ & $\frac{\gamma}{2 \mu \gamma+1}\left(\mathrm{~W}\left(\frac{2 \mu \gamma+1}{\gamma} \exp \left(\frac{\lambda_{i}}{\gamma}-1\right)\right)\right)_{1 \leq i \leq n}$ \\
\hline $\begin{array}{l}\text { Schatten } p \text {-penalty } \\
\mu \mathcal{R}_{p}^{p}(\mathbf{C}), p \geq 1\end{array}$ & $\begin{array}{c}\left(d_{i}\right)_{1 \leq i \leq n} \\
\text { with }(\forall i \in\{1, \ldots, n\}) d_{i}>0 \text { and } p \mu \gamma d_{i}^{p-1}+d_{i}+\gamma \log d_{i}+\gamma=\lambda_{i}\end{array}$ \\
\hline $\begin{array}{l}\text { Bounds on eigenvalues } \\
\qquad \iota_{E_{2}}(\mathbf{C})\end{array}$ & $\left(\min \left(\max \left(\gamma \mathrm{W}\left(\frac{1}{\gamma} \exp \left(\frac{\lambda_{i}}{\gamma}-1\right)\right), \alpha\right), \beta\right)\right)_{1 \leq i \leq n},[\alpha, \beta] \subset[0,+\infty]$ \\
\hline $\begin{array}{c}\text { Rank } \\
\mu \operatorname{rank}(\mathbf{C})\end{array}$ & $(\forall i \in\{1, \ldots, n\}) d_{i}=\left\{\begin{array}{ll}\rho_{i} & \left.\text { if } d_{i}\right)_{1 \leq i \leq n} \text { with } \\
0 \text { or } \rho_{i} & \text { if } \rho_{i}=\chi \\
0 & \text { otherwise }\end{array}\right.$ and $\left\{\begin{array}{l}\chi=\sqrt{\gamma(\gamma+2 \mu)}-\gamma, \\
\rho_{i}=\gamma \mathrm{W}\left(\frac{1}{\gamma} \exp \left(\frac{\lambda_{i}}{\gamma}-1\right)\right)\end{array}\right.$ \\
\hline
\end{tabular}

\subsection{Douglas-Rachford Algorithm}

We now propose a Douglas-Rachford (DR) approach ([57, 46, 58, ) for numerically solving Problem (1). We point out that the DR algorithm is directly related to the Alternating Direction Method of Multipliers $(\mathrm{ADMM})$, since the latter can be viewed as a version of the former applied to a dual formulation of the problem. The DR method minimizes the sum of $f-\operatorname{trace}(\mathbf{T} \cdot)+g_{0}$ and $g_{1}$ by alternately computing proximity operators of each of these functions. Proposition 2 allows us to calculate the proximity operator of $\gamma\left(f-\operatorname{trace}(\mathbf{T} \cdot)+g_{0}\right)$ with $\left.\gamma \in\right] 0,+\infty[$, by possibly using the expressions listed in Tables 1, 2, and 3 , Since $g_{1}$ is not a spectral function, $\operatorname{prox}_{\gamma g_{1}}$ has to be derived from other expressions of proximity operators. For instance, if $g_{1}$ is a separable sum of functions of its elements, e.g. $g=\|\cdot\|_{1}$, standard expressions for the proximity operator of vector functions can be employed [51, 46, 11

The computations to be performed are summarized in Algorithm 1 . We state a convergence theorem in the matrix framework, which is an offspring of existing results in arbitrary Hilbert spaces (see, for example, [46] and [59, Proposition 3.5]).

Theorem 3. Let $f$ and $g_{0}$ be functions satisfying (4) and (5), respectively, where $\varphi \in \Gamma_{0}\left(\mathbb{R}^{n}\right)$ and $\psi \in$ $\Gamma_{0}\left(\mathbb{R}^{n}\right)$. Let $g_{1} \in \Gamma_{0}\left(\mathcal{S}_{n}\right)$ be such that $f-\operatorname{trace}(\mathbf{T} \cdot)+g_{0}+g_{1}$ is coercive. Assume that the intersection of the relative interiors of the domains of $f+g_{0}$ and $g_{1}$ is non empty. Let $\left(\alpha^{(k)}\right)_{k \geq 0}$ be a sequence in $[0,2]$ such that $\sum_{k=0}^{+\infty} \alpha^{(k)}\left(2-\alpha^{(k)}\right)=+\infty$. Then, the sequences $\left(\mathbf{C}^{\left(k+\frac{1}{2}\right)}\right)_{k \geq 0}$ and $\left(\operatorname{prox}_{\gamma g_{1}}\left(2 \mathbf{C}^{\left(k+\frac{1}{2}\right)}-\mathbf{C}^{(k)}\right)\right)_{k \geq 0}$ generated by Algorithm 1 converge to a solution to Problem (1) where $g=g_{0}+g_{1}$.

\footnotetext{
${ }^{1}$ See also http://proximity-operator.net.
} 


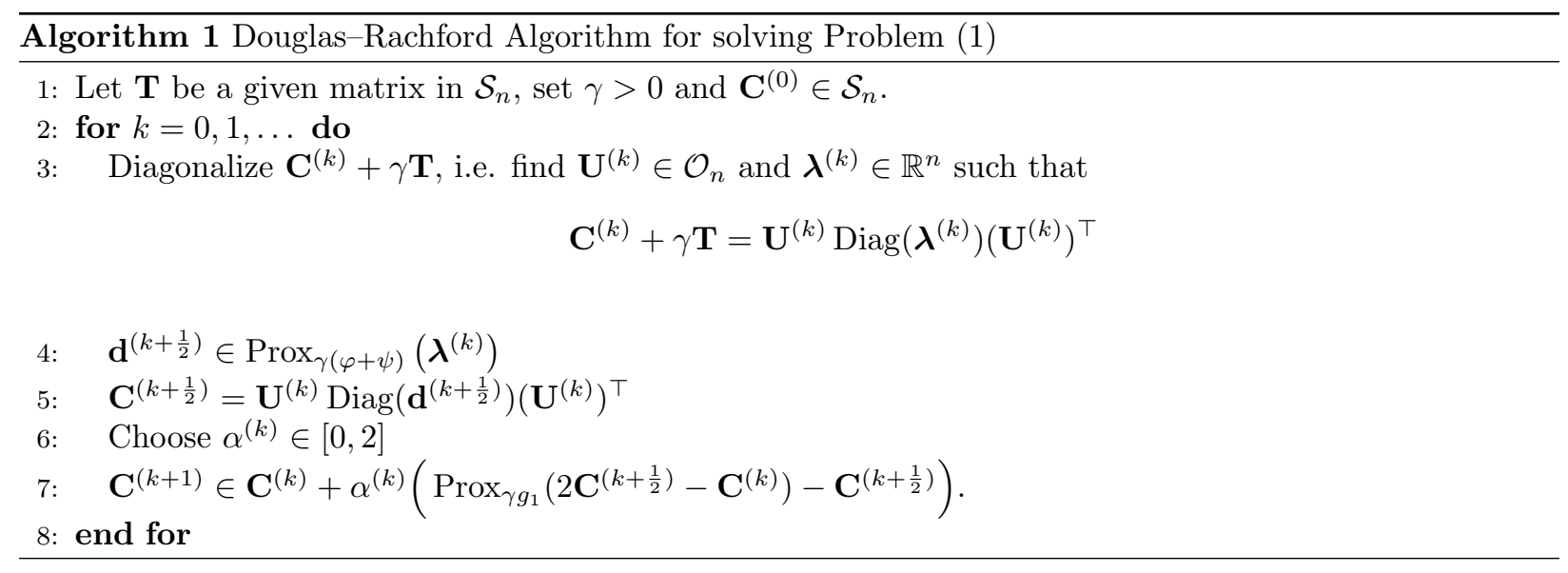

We have restricted the above convergence analysis to the convex case. Note however that recent convergence results for the DR algorithm in a non-convex setting are available in [60,61 for specific choices of the involved functionals.

\subsection{Positive Semi-Definite Constraint}

Instead of solving Problem (1D, one may be interested in:

$$
\underset{\mathbf{C} \in \mathcal{S}_{n}^{+}}{\operatorname{minimize}} f(\mathbf{C})-\operatorname{trace}(\mathbf{C T})+g(\mathbf{C})
$$

when $\operatorname{dom} f \cap \operatorname{dom} g \not \subset \mathcal{S}_{n}^{+}$. This problem can be recast as minimizing over $\mathcal{S}_{n} f$-trace $(\cdot \mathbf{T})+\widetilde{g}_{0}+g_{1}$ where $\widetilde{g}_{0}=g_{0}+\iota_{\mathcal{S}_{n}^{+}}$. We are thus coming back to the original formulation where $\widetilde{g}_{0}$ has been substituted for $g_{0}$. In order to solve this problem with the proposed proximal approach, a useful result is stated below.

Theorem 4. Let $\gamma \in] 0,+\infty\left[\right.$ and $\overline{\mathbf{C}} \in \mathcal{S}_{n}$. Let $f$ and $g_{0}$ be functions satisfying (4) and (5), respectively, where $\varphi \in \Gamma_{0}\left(\mathbb{R}^{n}\right)$ and $\psi \in \Gamma_{0}\left(\mathbb{R}^{n}\right)$. Assume that

$$
\left(\forall \boldsymbol{\lambda}^{\prime}=\left(\lambda_{i}^{\prime}\right)_{1 \leq i \leq n} \in \mathbb{R}^{n}\right) \quad \varphi\left(\boldsymbol{\lambda}^{\prime}\right)+\psi\left(\boldsymbol{\lambda}^{\prime}\right)=\sum_{i=1}^{n} \rho_{i}\left(\lambda_{i}^{\prime}\right)
$$

where, for every $\left.\left.i \in\{1, \ldots, n\}, \rho_{i}: \mathbb{R} \rightarrow\right]-\infty,+\infty\right]$ is such that $\operatorname{dom} \rho_{i} \cap\left[0,+\infty\left[\neq \varnothing\right.\right.$. Let $\boldsymbol{\lambda}=\left(\lambda_{i}\right)_{1 \leq i \leq n} \in \mathbb{R}^{n}$ and $\mathbf{U} \in \mathcal{O}_{n}$ be such that $\overline{\mathbf{C}}+\gamma \mathbf{T}=\mathbf{U} \operatorname{Diag}(\boldsymbol{\lambda}) \mathbf{U}^{\top}$. Then

$$
\operatorname{prox}_{\gamma\left(f-\operatorname{trace}(\mathbf{T} \cdot)+\widetilde{g}_{0}\right)}(\overline{\mathbf{C}})=\mathbf{U} \operatorname{Diag}\left(\left(\max \left(0, \operatorname{prox}_{\gamma \rho_{i}}\left(\lambda_{i}\right)\right)\right)_{1 \leq i \leq n}\right) \mathbf{U}^{\top}
$$

Proof. Expression 25) readily follows from Theorem 2(ii) and [62, Proposition 2.2].

\section{Robust Estimation in Gaussian Graphical Models}

Estimating the covariance matrix of a random vector is a key problem in statistics, signal processing, and machine learning [17, 18, 8, 6, 63. A related problem can be found in graphical modeling: in this case, 
the problem consists of estimating the graph adjacency matrix, which is modeled as the precision matrix (i.e., the inverse of covariance matrix) of the random Gaussian vector associated with the nodes of the graph. Nonetheless, in existing techniques devoted to solve the aforementioned problems, little attention is usually paid to the presence of noise corrupting the available observations. We develop in this section two novel formulations which account for noise information. Firstly, we address the problem of covariance matrix estimation. The chosen objective function consists of a squared Frobenius norm term coupled with regularization functions driven by the targeted application, and it can be minimized efficiently by using our DR method. The second problem is the estimation of the precision matrix under sparsity constraints. To the best of our knowledge, no method is available in the literature to solve the non-convex problem arising in this case. Here, we propose to resort to a majorization-minimization (MM) strategy, combined with the previously described DR procedure. Note that a different MM formulation for estimating sparse covariance matrices was proposed in the seminal work in 9 .

\subsection{Models and Proposed Approaches}

Let $\mathbf{S} \in \mathcal{S}_{n}^{+}$be a sample estimate of a covariance matrix $\mathbf{\Sigma}$ which is assumed to be decomposed as

$$
\boldsymbol{\Sigma}=\mathbf{Y}^{*}+\sigma^{2} \mathrm{I}_{\mathrm{d}}
$$

where $\sigma \in\left[0,+\infty\left[\right.\right.$ and $\mathbf{Y}^{*} \in \mathcal{S}_{n}^{+}$may have a low-rank structure. We focus on the problem of searching an estimate of $\mathbf{Y}^{*}$ from $\mathbf{S}$ by assuming that $\sigma$ is known. More specifically, we consider the following observation model [64]:

$$
(\forall i \in\{1, \ldots, N\}) \quad \mathbf{x}^{(i)}=\mathbf{A} \mathbf{s}^{(i)}+\mathbf{e}^{(i)}
$$

where $\mathbf{A} \in \mathbb{R}^{n \times m}$ with $m \leq n$ and, for every $i \in\{1, \ldots, N\}, \mathbf{s}^{(i)} \in \mathbb{R}^{m}$ and $\mathbf{e}^{(i)} \in \mathbb{R}^{n}$ are realizations of mutually independent identically distributed Gaussian multivalued random variables with zero mean and covariance matrices $\mathbf{P} \in \mathcal{S}_{m}^{++}$and $\sigma^{2} I_{\mathrm{d}}$, respectively. The latter model has been employed for instance in [65, 66] in the context of the "Relevant Vector Machine problem". The covariance matrix $\boldsymbol{\Sigma}$ of the noisy input data $\left(\mathbf{x}^{(i)}\right)_{1 \leq i \leq N}$ takes the form 26 with $\mathbf{Y}^{*}=\mathbf{A P A} \mathbf{A}^{\top}$. A rough estimate of $\boldsymbol{\Sigma}$ from the observed data $\left(\mathbf{x}^{(i)}\right)_{1 \leq i \leq N}$ can be obtained through the empirical covariance:

$$
\mathbf{S}=\frac{1}{N} \sum_{i=1}^{N} \mathbf{x}^{(i)}\left(\mathbf{x}^{(i)}\right)^{\top}
$$

In the following, we propose two alternative variational approaches for the estimation of $\boldsymbol{\Sigma}$ given the noisy input data $\left(\mathbf{x}^{(i)}\right)_{1 \leq i \leq N}$.

Covariance-based model. Our first formulation yields an estimate $\widehat{\mathbf{Y}}$ of $\mathbf{Y}^{*}$ given by

$$
\widehat{\mathbf{Y}}=\underset{\mathbf{Y} \in \mathcal{S}_{n}^{+}}{\operatorname{argmin}} \frac{1}{2}\left\|\mathbf{Y}-\left(\mathbf{S}+\sigma^{2} \mathrm{I}_{\mathrm{d}}\right)\right\|_{\mathrm{F}}^{2}+g_{0}(\mathbf{Y})+g_{1}(\mathbf{Y}),
$$


where $\mathbf{S}$ is the empirical covariance matrix, $g_{0}$ satisfies $[5]$ with $\psi \in \Gamma_{0}\left(\mathbb{R}^{n}\right), g_{1} \in \Gamma_{0}\left(\mathcal{S}_{n}\right)$, and the intersection of the relative interiors of the domains of $g_{0}$ and $g_{1}$ is assumed to be non empty. A particular instance of this model with $\sigma=0, g_{0}=\mu_{0} \mathcal{R}_{1}, g_{1}=\mu_{1}\|\cdot\|_{1}$, and $\left(\mu_{0}, \mu_{1}\right) \in\left[0,+\infty{ }^{2}\right.$ was investigated in [39] and [40] for estimating sparse low-rank covariance matrices. In the latter reference, an application to real data processing arising from protein interaction and social network analysis was presented. One can observe that Problem 29p takes the form 23 by setting $f=\frac{1}{2}\|\cdot\|_{\mathrm{F}}^{2}$ and $\mathbf{T}=\mathbf{S}-\sigma^{2} \mathrm{I}_{\mathrm{d}}$. This allows us to solve 29p with Algorithm 1. Since $g_{0}$ is assumed to satisfy (5), the proximity step on $f+g_{0}+\iota_{\mathcal{S}_{n}^{+}}$can be performed by employing Theorem 4 and formulas from Table 1 . The resulting DR procedure can thus be viewed as an alternative to the methods developed in [40] and [39. Let us emphasize that these two algorithms were devised to solve an instance of 29 corresponding to the aforementioned specific choices for $g_{0}$ and $g_{1}$, while our approach leaves more freedom in the choice of the regularization functions. A comparison of the three algorithms will be performed in Section 5 .

Precision-based model. Our second strategy focuses on the estimation of the inverse of the covariance matrix, i.e. the precision matrix $\mathbf{C}^{*}=\left(\mathbf{Y}^{*}\right)^{-1}$ by assuming that $\mathbf{Y}^{*} \in \mathcal{S}_{n}^{++}$but may have very small eigenvalues in order to model a possible low-rank structure. Tackling the problem from this viewpoint leads us to propose the following penalized negative log-likelihood cost function:

$$
\left(\forall \mathbf{C} \in \mathcal{S}_{n}\right) \quad \mathcal{F}(\mathbf{C})=f(\mathbf{C})+\mathcal{T}_{\mathbf{S}}(\mathbf{C})+g_{0}(\mathbf{C})+g_{1}(\mathbf{C})
$$

where

$$
\begin{array}{r}
\left(\forall \mathbf{C} \in \mathcal{S}_{n}\right) \quad f(\mathbf{C})= \begin{cases}\log \operatorname{det}\left(\mathbf{C}^{-1}+\sigma^{2} \mathrm{I}_{\mathrm{d}}\right) & \text { if } \mathbf{C} \in \mathcal{S}_{n}^{++} \\
+\infty & \text { otherwise }\end{cases} \\
\left(\forall \mathbf{C} \in \mathcal{S}_{n}\right) \quad \mathcal{T}_{\mathbf{S}}(\mathbf{C})= \begin{cases}\operatorname{trace}\left(\left(\mathrm{I}_{\mathrm{d}}+\sigma^{2} \mathbf{C}\right)^{-1} \mathbf{C S}\right) & \text { if } \mathbf{C} \in \mathcal{S}_{n}^{+} \\
+\infty & \text { otherwise }\end{cases}
\end{array}
$$

$g_{0} \in \Gamma_{0}\left(\mathcal{S}_{n}\right)$ satisfies (5) with $\psi \in \Gamma_{0}\left(\mathbb{R}^{n}\right)$, and $g_{1} \in \Gamma_{0}\left(\mathcal{S}_{n}\right)$. Typical choices of interest for the latter two functions are

$$
\left(\forall \mathbf{C} \in \mathcal{S}_{n}\right) \quad g_{0}(\mathbf{C})= \begin{cases}\mu_{0} \mathcal{R}_{1}\left(\mathbf{C}^{-1}\right) & \text { if } \mathbf{C} \in \mathcal{S}_{n}^{++} \\ +\infty & \text { otherwise }\end{cases}
$$

and $g_{1}=\mu_{1}\|\cdot\|_{1}$ with $\left(\mu_{0}, \mu_{1}\right) \in\left[0,+\infty{ }^{2}\right.$. The first function serves to promote a desired low-rank property by penalizing small eigenvalues of the precision matrix, whereas the second one enforces the sparsity of this matrix as it is usual in graph inference problems. Note that the standard graphical lasso framework [6] is then recovered by setting $\sigma=0$ and $\mu_{0}=0$. The advantage of our formulation is that it allows us to consider more flexible variational models while accounting for the presence of noise corrupting the observed 
data. The main difficulty however is that Algorithm 1 (or its dual counterpart ADMM) cannot be directly applied to minimize the non-convex cost $\mathcal{F}$. In Section 4.2, we study in more details the properties of the latter cost function. This leads us to derive a novel optimization algorithm based on the MM principle, making use of our previously developed Douglas-Rachford scheme for its inner steps

\subsection{Study of Objective Function $\mathcal{F}$}

The following lemma will reveal useful in our subsequent analysis.

Lemma 1. Let $\sigma \in] 0,+\infty[$. Let $h:] 0, \sigma^{-2}[\rightarrow \mathbb{R}$ be a twice differentiable function and let

$$
u:\left[0,+\infty\left[\rightarrow \mathbb{R}: \lambda \mapsto \frac{\lambda}{1+\sigma^{2} \lambda} .\right.\right.
$$

The composition $h \circ u$ is convex on $] 0,+\infty[$ if and only if

$$
(\forall v \in] 0, \sigma^{-2}[) \quad \ddot{h}(v)\left(1-\sigma^{2} v\right)-2 \sigma^{2} \dot{h}(v) \geq 0,
$$

where $\dot{h}$ (resp. $\ddot{h}$ ) denotes the first (resp. second) derivative of $h$.

Proof. The result directly follows from the calculation of the second-order derivative of $h \circ u$.

Let us now note that $f$ is a spectral function fulfilling (4) with

$$
\left(\forall \boldsymbol{\lambda}=\left(\lambda_{i}\right)_{1 \leq i \leq n} \in \mathbb{R}^{n}\right) \quad \varphi(\boldsymbol{\lambda})= \begin{cases}-\sum_{i=1}^{n} \log \left(u\left(\lambda_{i}\right)\right) & \text { if } \boldsymbol{\lambda} \in] 0,+\infty\left[^{n}\right. \\ +\infty & \text { otherwise }\end{cases}
$$

where $u$ is defined by (34). According to Lemma 1 (with $h=-\log$ ), $f \in \Gamma_{0}\left(\mathcal{S}_{n}\right)$. Thus, the assumptions made on $g_{0}$ and $g_{1}$, allow us to deduce that $f+g_{0}+g_{1}$ is convex and lower-semicontinuous on $\mathcal{S}_{n}$.

Let us now focus on the properties of the second term in 30 .

Lemma 2. Let $\mathbf{S} \in \mathcal{S}_{n}^{+}$. The function $\mathcal{T}_{\mathbf{S}}$ in 32 is concave on $\mathcal{S}_{n}^{+}$.

Proof. See Appendix B

As a last worth mentioning property, $\mathcal{T}_{\mathbf{S}}$ is bounded on $\mathcal{S}_{n}^{++}$. So, if $\operatorname{dom} f \cap \operatorname{dom} g_{0} \cap \operatorname{dom} g_{1} \neq \varnothing$ and $f+g_{0}+g_{1}$ is coercive, then there exists a minimizer of $\mathcal{F}$. Because of the form of $f$, the coercivity condition is satisfied if $g_{0}+g_{1}$ is lower bounded and $\lim _{\mathbf{C} \in \mathcal{S}_{n}^{+},\|\mathbf{C}\| \rightarrow+\infty} g_{0}(\mathbf{C})+g_{1}(\mathbf{C})=+\infty$.

\subsection{Minimization Algorithm for $\mathcal{F}$}

In order to find a minimizer of $\mathcal{F}$, we propose a Majorize-Minimize (MM) approach, following the ideas in 67, 64, 68, 69, 70, 71]. At each iteration of an MM algorithm, one constructs a tangent function that majorizes the given cost function and is equal to it at the current iterate. The next iterate is obtained by 
minimizing this tangent majorant function, resulting in a sequence of iterates that reduces the cost function value monotonically. According to the results stated in the previous section, our objective function reads as a difference of convex terms. We propose to build a majorizing approximation of function $\mathcal{T}_{\mathbf{S}}$ at $\mathbf{C}^{\prime} \in \mathcal{S}_{n}^{++}$ by exploiting Lemma 2 and the classical concavity inequality on $\mathcal{T}_{\mathbf{S}}$ :

$$
\left(\forall \mathbf{C} \in \mathcal{S}_{n}^{++}\right) \quad \mathcal{T}_{\mathbf{S}}(\mathbf{C}) \leq \mathcal{T}_{\mathbf{S}}\left(\mathbf{C}^{\prime}\right)+\operatorname{trace}\left(\nabla \mathcal{T}_{\mathbf{S}}\left(\mathbf{C}^{\prime}\right)\left(\mathbf{C}-\mathbf{C}^{\prime}\right)\right) .
$$

As $f$ is finite only on $\mathcal{S}_{n}^{++}$, a tangent majorant of the cost function 30 at $\mathbf{C}^{\prime}$ reads:

$$
\left(\forall \mathbf{C} \in \mathcal{S}_{n}\right) \quad \mathcal{G}\left(\mathbf{C} \mid \mathbf{C}^{\prime}\right)=f(\mathbf{C})+\mathcal{T}_{\mathbf{S}}\left(\mathbf{C}^{\prime}\right)+\operatorname{trace}\left(\nabla \mathcal{T}_{\mathbf{S}}\left(\mathbf{C}^{\prime}\right)\left(\mathbf{C}-\mathbf{C}^{\prime}\right)\right)+g_{0}(\mathbf{C})+g_{1}(\mathbf{C}) .
$$

This leads to the general MM scheme:

$$
(\forall \ell \in \mathbb{N}) \quad \mathbf{C}^{(\ell+1)} \in \underset{\mathbf{C} \in \mathcal{S}_{n}}{\operatorname{argmin}} f(\mathbf{C})+\operatorname{trace}\left(\nabla \mathcal{T}_{\mathbf{S}}\left(\mathbf{C}^{(\ell)}\right) \mathbf{C}\right)+g_{0}(\mathbf{C})+g_{1}(\mathbf{C})
$$

with $\mathbf{C}^{(0)} \in \mathcal{S}_{n}^{++}$. At each iteration of the MM algorithm, we have then to solve a convex optimization problem of the form (1). In the case when $g_{1} \equiv 0$, we can employ the procedure described in Section 2 to perform this task in a direct manner. The presence of a regularization term $g_{1} \not \equiv 0$ usually prevents us to have an explicit solution to the inner minimization problem involved in the MM procedure. We then propose in Algorithm 2 to resort to the Douglas-Rachford approach in Section 3 to solve it iteratively. A convergence result is next stated, which is inspired from [72] (itself relying on [73, p. 6]), but does not require the differentiability of $g_{0}+g_{1}$.

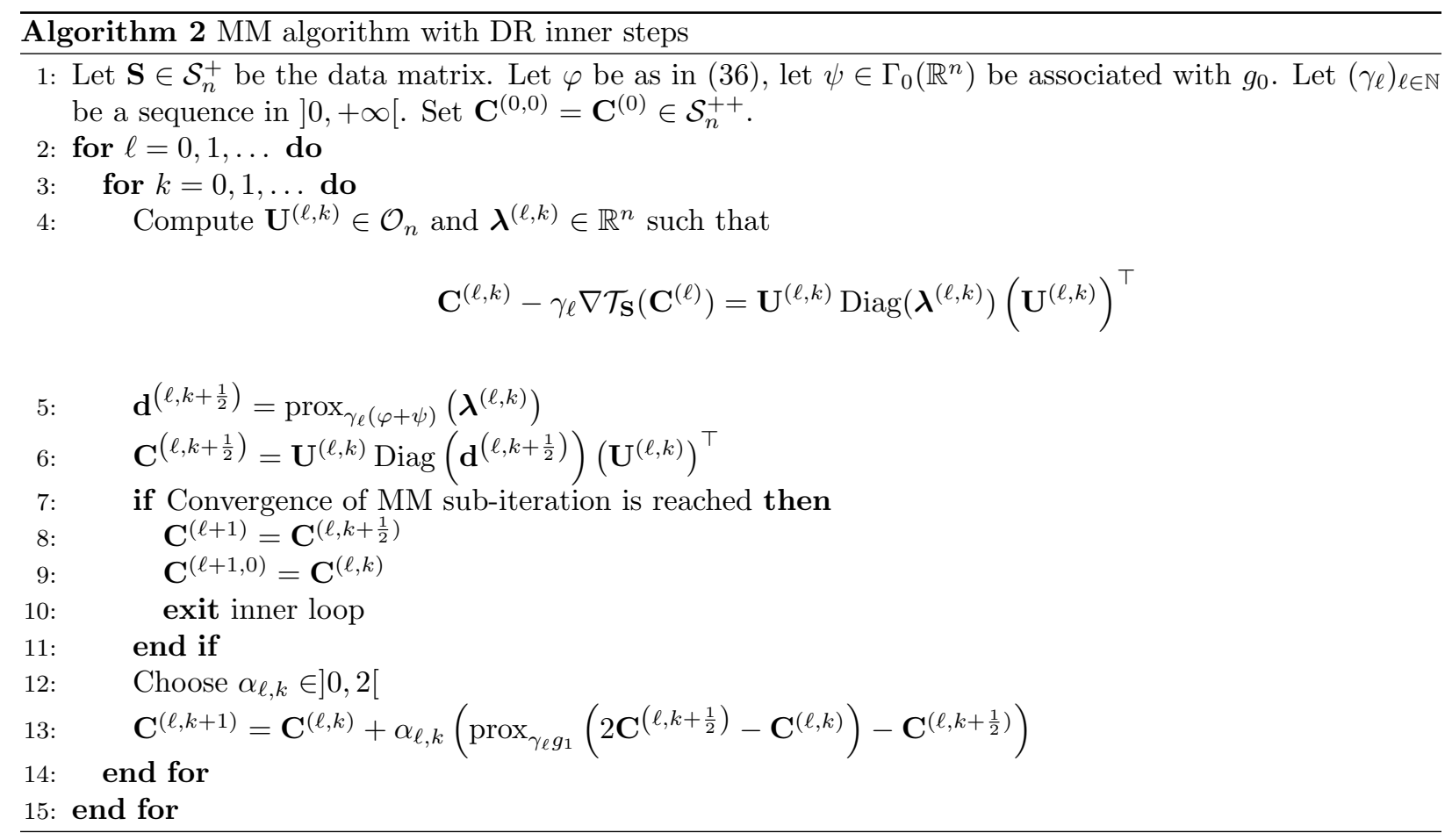


Theorem 5. Let $\quad\left(\mathbf{C}^{(\ell)}\right)_{\ell \geq 0}$ be a sequence generated by 38. Assume that $\operatorname{dom} f \cap \operatorname{dom} g_{0} \cap \operatorname{dom} g_{1} \neq \varnothing, f+g_{0}+g_{1}$ is coercive, and $E=\left\{\mathbf{C} \in \mathcal{S}_{n} \mid \mathcal{F}(\mathbf{C}) \leq \mathcal{F}\left(\mathbf{C}^{(0)}\right)\right\}$ is a subset of the relative interior of $\operatorname{dom} g_{0} \cap \operatorname{dom} g_{1}$. Then, the following properties hold:

(i) $\left(\mathcal{F}\left(\mathbf{C}^{(\ell)}\right)\right)_{\ell \geq 0}$ is a decaying sequence converging to $\widehat{\mathcal{F}} \in \mathbb{R}$.

(ii) $\left(\mathbf{C}^{(\ell)}\right)_{\ell \geq 0}$ has a cluster point.

(iii) Every cluster point $\widehat{\mathbf{C}}$ of $\left(\mathbf{C}^{(\ell)}\right)_{\ell \geq 0}$ is such that $\mathcal{F}(\widehat{\mathbf{C}})=\widehat{\mathcal{F}}$ and it is a critical point of $\mathcal{F}$, i.e. $-\nabla f(\widehat{\mathbf{C}})-$ $\nabla \mathcal{T}_{\mathbf{S}}(\widehat{\mathbf{C}}) \in \partial\left(g_{0}+g_{1}\right)(\widehat{\mathbf{C}})$

Proof. See Appendix C.

\section{Numerical Experiments}

This section presents some numerical tests illustrating the validity of the proposed algorithms. All the tests were ran on a Hewlett-Packard Notebook with 16GB or RAM, INTEL i5 CPU (1.6GHz) equipped with MatLab R2019a. All the numerical tests are reproducible, using the code we made available at http:

\section{//www-syscom.univ-mlv.fr/ benfenat/Software.html}

\subsection{Application to Sparse Covariance Matrix Estimation}

We first consider the application of the DR algorithm from Section 3 to the sparse covariance matrix estimation problem introduced in [40. As we have shown in Section 4.1, a solution to this problem can be obtained by solving the penalized least-squares problem 29 , where $\mathbf{S}$ is the empirical covariance matrix defined in (28), and the regularization terms are $g_{0}=\mu_{0} \mathcal{R}_{1}$ and $g_{1}=\mu_{1}\|\cdot\|_{1}$. We propose to compare the performance of the DR approach from Section 3.2. with the IPD algorithm [40] and the ADMM procedure [39], for solving this convex optimization problem. The synthetic data are generated using a procedure similar to the one in [40]. A block-diagonal covariance matrix $\mathbf{Y}^{*}$ is considered, composed of $r$ blocks with dimensions $\left(r_{j}\right)_{1 \leq j \leq r}$, so that $n=\sum_{j=1}^{r} r_{j}$. The $j$-th diagonal block of $\mathbf{Y}^{*}$ reads as a product $\mathbf{a}_{j} \mathbf{a}_{j}^{\top}$, where the components of $\mathbf{a}_{j} \in \mathbb{R}^{r_{j}}$ are randomly drawn on $[-1,1]$. The number of observations $N$ is set equal to $n$ with $n \in\{100,300,500,1000\}$ and, for each dimension $n$, we consider 10 noise realizations with standard deviation $\sigma=0.1$. The three tested algorithms are initialized with $\mathbf{S}+\mathrm{I}_{\mathrm{d}}$, and stopped as soon as a relative decrease criterion on the objective function is met, i.e. when $\left|\mathcal{F}_{k+1}-\mathcal{F}\right| \leq \varepsilon\left|\mathcal{F}_{k}\right|, \varepsilon>0$ being a given tolerance and $\mathcal{F}_{k}$ denoting the objective function value at iteration $k$. The maximum number of iterations is set to 3000. The gradient stepsize for IPD is set to $k^{-1}$. In Algorithm $1, \alpha_{k}$ is set to 1.5. In ADMM, the initial Lagrange multiplier is set to a matrix with all entries equal to one, and the parameter of the proximal step is set to 1 .

Fig. 1 illustrates the quality of the recovered covariance matrices (for $n=100$ or 500) when setting $\varepsilon=10^{-10}$ and for one out of ten noise realizations. Three different indicators for estimation quality are 

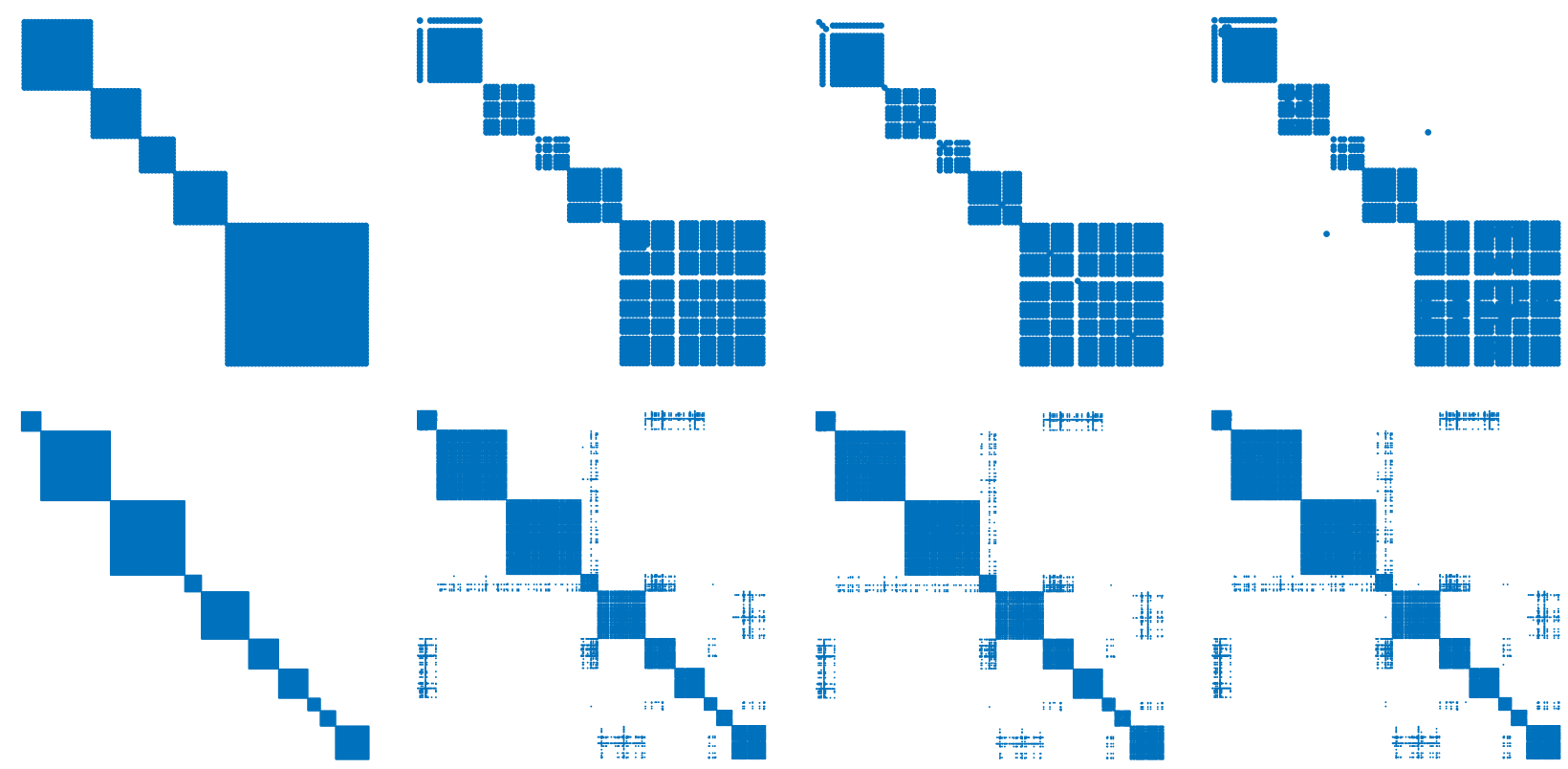

Figure 1: Original matrix and reconstruction results for DR, ADMM and IPD algorithms, for $n=100$ (top) and $n=300$ (bottom).

provided, namely the true positive rate (tpr), i.e. the correctly recognized non-zero entries, the false positive rate $(\mathrm{fpr})$, i.e. the entries erroneously added to the support of the matrix, and the relative mean square error (rmse), computed as $\left\|\mathbf{Y}_{\text {rec }}-\mathbf{Y}^{*}\right\|_{\mathrm{F}}^{2} /\left\|\mathbf{Y}^{*}\right\|_{\mathrm{F}}^{2}$, where $\mathbf{Y}_{\text {rec }}$ is the recovered matrix. The penalty parameters $\mu_{1}$ and $\mu_{0}$ are chosen empirically so as to minimize rmse on a single noise realization. Note that the two first measurements are employed when the main interest lies in the recovery of the matrix support. A visual inspection shows that the three methods provide similar results in terms of matrix support estimation. The numerical values of the 3 indicators are depicted in Table 4 showing that the three methods achieve similar quantitative scores.

Table 4: Numerical results for $\varepsilon=10^{-10}$, averaged over 10 different noise realizations. All the algorithms provide similar results, in terms of rmse, fpr and tpr, for each test case.

\begin{tabular}{|c|c|c|c|c|c|c|c|c|c|}
\hline$n$ & & $\mathrm{DR}$ & ADMM & IPD & $n$ & & $\mathrm{DR}$ & ADMM & IPD \\
\hline \multirow{3}{*}{100} & rmse & 0.3715 & 0.3715 & 0.3778 & \multirow{3}{*}{500} & rmse & 0.2654 & 0.2825 & 0.2691 \\
\hline & tpr & $72.29 \%$ & $73.27 \%$ & $75.14 \%$ & & tpr & $74.97 \%$ & $74.85 \%$ & $76.98 \%$ \\
\hline & fpr & $1.65 \%$ & $1.55 \%$ & $1.54 \%$ & & fpr & $0.21 \%$ & $0.25 \%$ & $0.26 \%$ \\
\hline \multirow{3}{*}{300} & rmse & 0.1849 & 0.1852 & 0.1828 & \multirow{3}{*}{1000} & rmse & 0.1707 & 0.1735 & 0.1799 \\
\hline & tpr & $80.81 \%$ & $81.17 \%$ & $82.78 \%$ & & tpr & $83.77 \%$ & $84.04 \%$ & $84.87 \%$ \\
\hline & fpr & $0.78 \%$ & $0.68 \%$ & $0.84 \%$ & & tpr & $0.14 \%$ & $0.21 \%$ & $0.24 \%$ \\
\hline
\end{tabular}


Table 5 allows us to compare the algorithms in terms of computation time (in seconds) and iteration number (averaged on 8 noise realizations, where the shortest and the longest times among the 10 runs were discarded), for the four scenarios corresponding to distinct problem sizes and block distributions. It can be observed that the behaviors of ADMM and DR are similar, while IPD requires many more iterations and time to reach the same precision. Furthermore, the latter fails to reach a high precision in the allowed maximum number of iterations, for all the four examples. The main source of computational cost of each procedure lies in the eigenvalue decomposition of a matrix, hence one iteration of any procedure among DR, ADMM and IPD takes approximately the same amount of time, which mainly depends on the matrix dimension. Furthermore, Table 5 shows that the DR approach often requires less iterations to achieve the same precision level, hence reaching a lower computational cost with respect to the other two procedures.

Table 5: Comparison in terms of convergence time between DR, ADMM and IPD procedures. The enlighten times refer to the shortest ones. Among the 10 realization, the shortest and the longest timings were discarded in the computation of the arithmetic mean.

\begin{tabular}{|c|c|c|c|c|c|c|}
\hline & \multicolumn{3}{|c|}{$\begin{array}{l}n=100, \mu_{0}=0.2, \mu_{1}=0.15, r=5 \\
\left\{r_{j}\right\}=\{20,14,10,15,41\}\end{array}$} & \multicolumn{3}{|c|}{$\begin{array}{l}n=300, \mu_{0}=0.1, \mu_{1}=0.1 \\
r=10,\left\{r_{j}\right\}=\{49,25,58,29,7,42,26,9,15,40\}\end{array}$} \\
\hline & DR & ADMM & IPD & $\mathrm{DR}$ & ADMM & IPD \\
\hline$\varepsilon$ & Time (iter) & Time (iter) & Time (iter) & Time(iter) & Time (iter) & Time (iter) \\
\hline $10^{-6}$ & $0.01 \quad(19)$ & $0.01 \quad(16)$ & 0.12 & $0.10 \quad(\mathbf{1 6})$ & $0.08 \quad(\mathbf{1 6})$ & $0.87 \quad(151)$ \\
\hline $10^{-7}$ & 00.02 & $0.04 \quad(60)$ & $0.37 \quad(581)$ & $0.19 \quad(31)$ & $0.30 \quad(48)$ & $3.01 \quad(484)$ \\
\hline $10^{-8}$ & 00.07 & $0.12(132)$ & $1.66(1867)$ & $0.30 \quad(47)$ & $0.76(114)$ & $10.02(1542)$ \\
\hline $10^{-9}$ & $00.13(146)$ & $0.26(281)$ & $2.62(3000)$ & $0.78(124)$ & $1.44(228)$ & $19.22(3000)$ \\
\hline \multirow[t]{2}{*}{$10^{-10}$} & $00.29(314)$ & $0.45(489)$ & $2.63(3000)$ & $1.52(238)$ & $2.37(371)$ & $18.92(3000)$ \\
\hline & \multicolumn{3}{|c|}{$\begin{array}{l}n=500, \mu_{0}=0.1, \mu_{1}=0.06, r=9 \\
\left\{r_{j}\right\}=\{132,112,72,3,24,1,43,30,83\}\end{array}$} & \multicolumn{3}{|c|}{$\begin{array}{l}n=1000, \mu_{0}=0.05, \mu_{1}=0.06, r=12 \\
\left\{r_{j}\right\}=\{189,171,59,58,7,120,64,34,19,86,60,133\}\end{array}$} \\
\hline $10^{-6}$ & $0.34 \quad(18)$ & $0.30(15)$ & $2.59 \quad(138)$ & $1.32 \quad(12)$ & $1.74 \quad(15)$ & $12.98(112)$ \\
\hline $10^{-7}$ & $1.06 \quad(51)$ & $1.60 \quad(77)$ & $8.90(446)$ & $3.15(24)$ & 6.11 & 44.21 \\
\hline $10^{-8}$ & $2.04 \quad(99)$ & $3.83(180)$ & $28.12(1431)$ & $6.66 \quad(54)$ & 11.01 & $139.43(1141)$ \\
\hline $10^{-9}$ & $4.47(208)$ & $9.13(411)$ & $60.93(3000)$ & $9.95(78)$ & $22.59(184)$ & $380.36(3000)$ \\
\hline $10^{-10}$ & $8.95(418)$ & $16.70(766)$ & $61.66(3000)$ & $18.56(139)$ & $48.91(357)$ & $387.83(3000)$ \\
\hline
\end{tabular}

\subsection{Application to Robust Graphical Lasso}

Let us now apply the MM approach presented in Section 4.3 to the problem of precision matrix estimation introduced in 30 on synthetic and real-world datasets.

Precision matrix estimation. A sparse precision matrix $\mathbf{C}^{*}$ of dimension $n \times n$ is randomly created, where the number of non-zero entries is chosen as a proportion $p \in] 0,1\left[\right.$ of the total number $n^{2}$. Then, $N$ realizations $\left(\mathbf{x}^{(i)}\right)_{1 \leq i \leq N}$ of a Gaussian multivalued random variable with zero mean and covariance $\mathbf{Y}^{*}=\left(\mathbf{C}^{*}\right)^{-1}$ are generated. Gaussian noise with zero mean and covariance $\sigma^{2} \mathrm{I}_{\mathrm{d}}, \sigma>0$, is finally added to the $\mathbf{x}^{(i)}$ 's, so that the covariance matrix $\boldsymbol{\Sigma}$ associated with the input data reads as in 26 with $\mathbf{A}=\mathrm{I}_{\mathrm{d}}$. As explained 


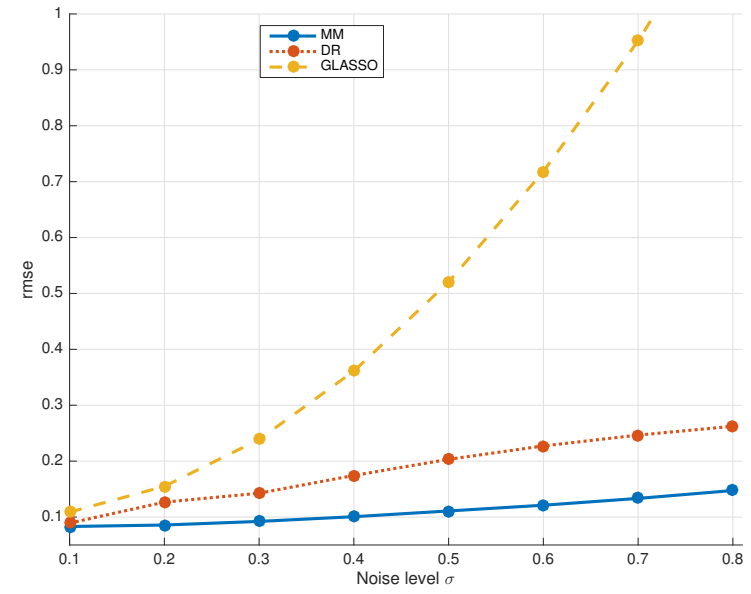

(a) Behaviour of rmse wrt $\sigma$.

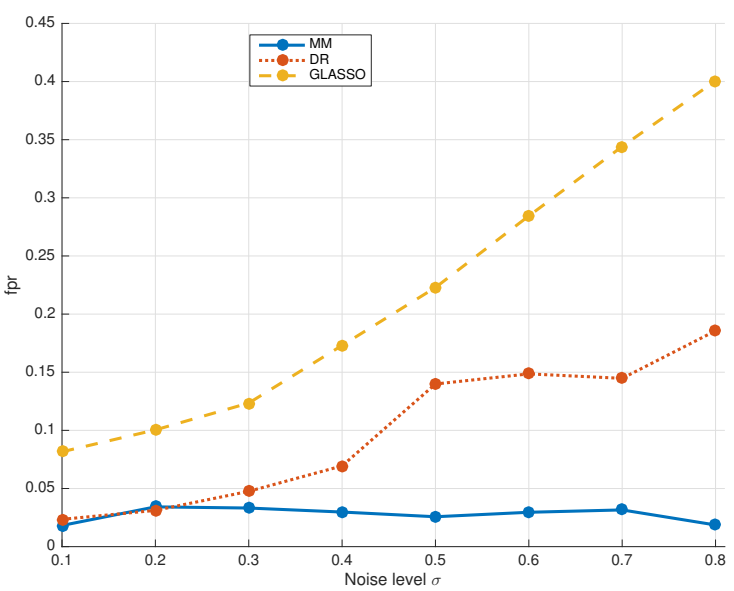

(b) Behaviour of fpr wrt $\sigma$.

Figure 2: Estimation results for different noise levels in terms of rmse (upper panel) and fpr (lower panel) for MM, GLASSO and DR approaches. The MM procedure has a stable behaviour wrt to increasing noise, while DR and GLASSO strongly suffer from the presence of noise.

in Section 4.1, the estimation of $\mathbf{C}^{*}$ can be performed by using the MM algorithm from Section 4.3 based on the minimization of the nonconvex cost $(30)$ with regularization functions $g_{1}=\mu_{1}\|\cdot\|_{1}, \mu_{1}>0$, and $\left(\forall \mathbf{C} \in \mathcal{S}_{n}^{++}\right) g_{0}(\mathbf{C})=\mu_{0} \mathcal{R}_{1}\left(\mathbf{C}^{-1}\right), \mu_{0}>0$. The computation of $\operatorname{prox}_{\gamma(\varphi+\psi)}$ with $\left.\gamma \in\right] 0,+\infty[$ related to this particular choice for $g_{0}$ and function $\varphi$ given by (36) and (34) leads to the search of the only positive root of a polynomial of degree 4 .

A synthetic dataset of size $n=100$ is created, where matrix $\mathbf{C}^{*}$ has 20 off-diagonal non-zero entries (i.e., $p=10^{-3}$ ) and the corresponding covariance matrix has condition number $0.125 . N=1000$ realizations are used to compute the empirical covariance matrix S. In our MM algorithm, the inner stopping criterion (line 7 in Algorithm 2 is based on the relative difference of majorant function values with a tolerance of $10^{-10}$, while the outer cycle is stopped when the relative difference of the objective function values falls below $10^{-8}$. The DR algorithm is used to solve the inner subproblems, by using parameters $(\forall \ell)$ $\gamma_{\ell}=1,(\forall k) \alpha_{\ell, k}=1$ (see Algorithm 2, lines 413 . The allowed maximum inner (resp. outer) iteration number is 2000 (resp. 20). The quality of the results is quantified in terms of $\mathrm{fpr}$ (false positive rate) on the precision matrix and rmse (relative mean square error) with respect to the true covariance matrix. The parameters $\mu_{1}$ and $\mu_{0}$ are set in order to obtain the best reconstruction in terms of rmse. For eight values of the noise standard deviation $\sigma$, Fig. 2 illustrates the reconstruction quality (averaged on 20 noise realizations) obtained with our method, as well as two convex minimization approaches that do not take into account the noise in their formulation, namely the classical GLASSO approach from [74, code available at http://stanford.edu/ boyd/papers/admm/covsel/covsel_example.html, which amounts to solve (1) with $f=-\log \operatorname{det}, g=\mu_{1}\|\cdot\|_{1}$, and the DR approach described in Section 3 , in the formulation given by 


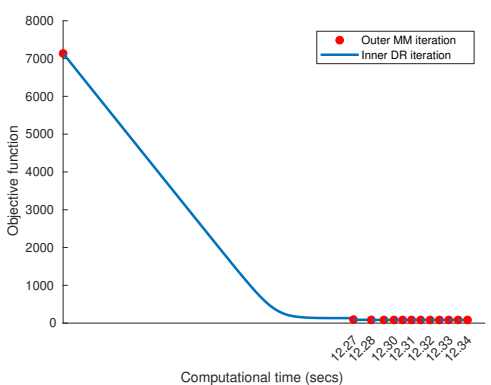

(a) MM

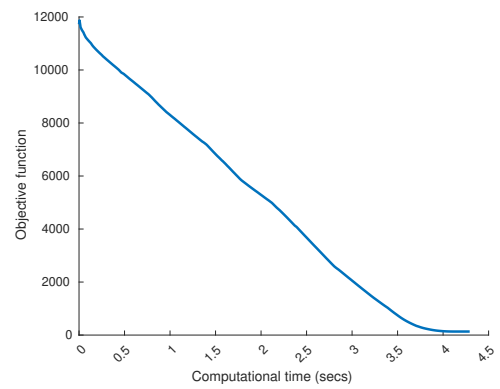

(b) DR

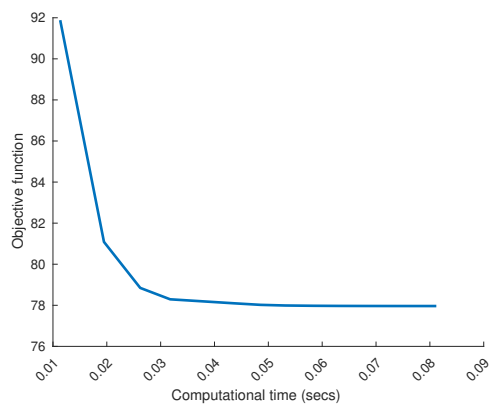

(c) GLASSO

Figure 3: Evolution of the objective functions of each tested method when $\sigma=0.5$. The GLASSO approach has the fastest rate, but on the other hand it provides no reliable results. The major cost of the MM procedure lies in the first iteration, while the other iterations are fast.

(1) with $f=-\log \operatorname{det},\left(\forall \mathbf{C} \in \mathcal{S}_{n}^{++}\right) g(\mathbf{C})=\mu_{0} \mathcal{R}_{1}\left(\mathbf{C}^{-1}\right)+\mu_{1}\|\mathbf{C}\|_{1}$. For the DR approach, $\operatorname{prox}_{\gamma(\varphi+\psi)}$ with $\gamma \in] 0,+\infty[$ is given by the fourth line of Table 2 (when $p=1$ ).

As expected, as the noise variance increases the reconstruction quality deteriorates. The GLASSO procedure is strongly impacted by the presence of noise, whereas the MM approach achieves better results, also when compared with DR algorithm. Moreover, the MM algorithm significantly outperforms both other methods in terms of support reconstruction, revealing itself very robust with respect to an increasing level of noise. Fig. 3 depicts the behavior of the objective function of each of the three compared methods, for the problem instance when $\sigma=0.5$, as a function of the computational time.

Molène Dataset. We now consider a real dataset consisting of weather information collected by 55 stations of Radome type located in a French region between $47^{\circ} \mathrm{N}$ and $49^{\circ} \mathrm{N}$, and $2^{\circ} \mathrm{W}$ and $6^{\circ} \mathrm{W}$. The data refer to the Archipel Molène project and they were collected from 1st Jan 2014 to 31st Jan 2014. They contain hourly information about rain (precipitation in $\mathrm{kg} / \mathrm{m}^{2}$ ), temperature (value, maximal temperature of air, minimal temperature of air) and wind (speed [10' mean], max speed [10' mean], max speed [m/s]; direction [10' mean], max direction [10' mean], max direction [angle]): this dataset is freely availabl $\phi^{2}$ a visualization of four snapshots of these data is shown in Fig. 4 . In this experiment the focus is on speed and direction of the wind: the collected data are stored in two matrices $\mathbf{W}_{d}$ and $\mathbf{W}_{s}$ both belonging to $\mathbb{R}^{31 \times 744}$, i.e. the hourly registrations $(744=24 \times 31$ ) were taken by 31 (over 55$)$ weather stations. The interest lies in finding connections between the different spots. The time interval considered in the whole dataset is quite large, it covers an entire month and then it can masquerade some interactions, so that only a subset referring to the first 3 days is retained. We also discarded the records from the weather station with code name PLOUDALMEZEAU as there were presenting erroneous and/or missing values. This pre-processing

\footnotetext{
2 https://www.data.gouv.fr/fr/datasets/donnees-horaires-des-55-stations-terrestres-de-la-zone-large-molenesur-un-mois/
} 


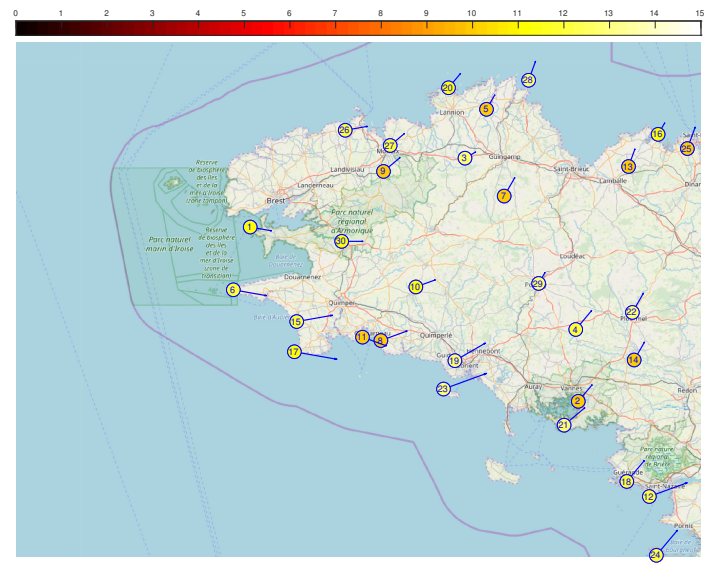

(a) $t=24($ day $2,00 \mathrm{H})$

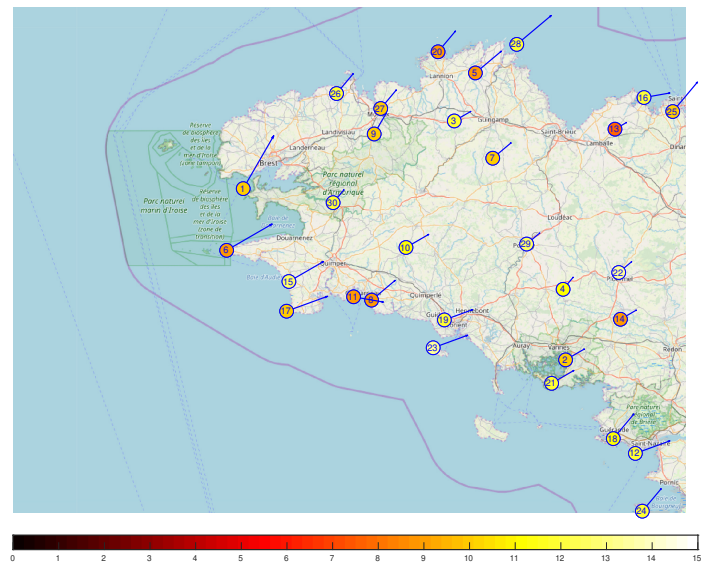

(c) $t=60($ day $3,12 \mathrm{H})$

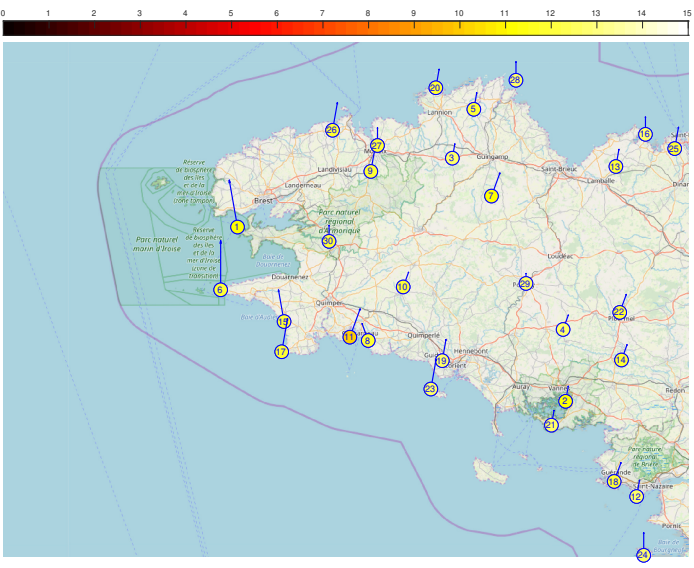

(b) $t=43($ day $2,19 \mathrm{H})$

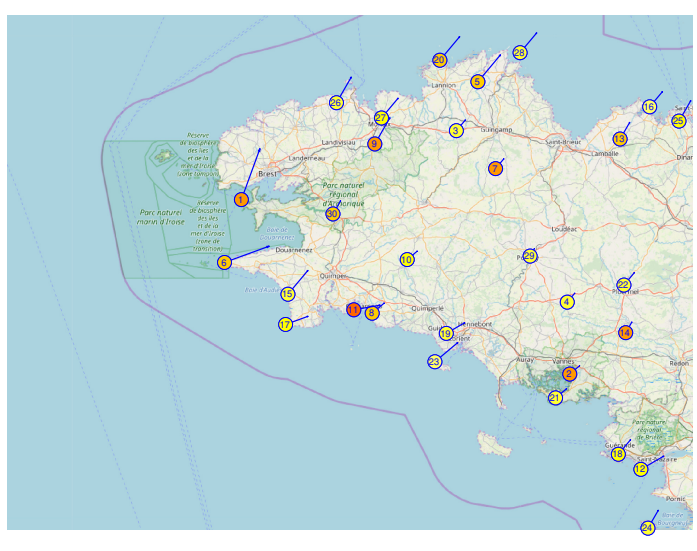

(d) $t=67($ day $3,19 \mathrm{H})$

Figure 4: Visualization of some snapshots of Molène Dataset. Each point represents a weather station (see ?? for stations' names). The blue arrows represent wind direction and their length is proportional to wind speed. The color of each station refers to the recorded temperature (in ${ }^{\circ} \mathrm{C}$ ). The map shows the French region between $47^{\circ} \mathrm{N}$ and $49^{\circ} \mathrm{N}$ and $2^{\circ} \mathrm{W}$ and $6^{\circ} \mathrm{W}$ and was downloaded from openstreetmap.org.

procedure leads to smaller matrices $\left(\mathbf{W}_{d}, \mathbf{W}_{s}\right) \in\left(\mathbb{R}^{30 \times 72}\right)^{2}$, i.e. $n=30$ and $N=72$. We propose to consider the wind data regarding both speed and direction in a coupled manner: a new data matrix $\mathbf{W}_{d s}=\mathbf{W}_{d} \odot \mathbf{W}_{s}$ is considered. In this way, the direction of the wind is modulated by its speed. Matrix $\mathbf{S}$ in Algorithm 2 is taken as $\mathbf{S}=\mathbf{D} \odot \mathbf{S}_{1}, \mathbf{S} \in \mathbb{R}^{30 \times 30}$, where $\mathbf{S}_{1}$ is the empirical covariance of the rows in $\mathbf{W}_{d s}$ and $\mathbf{D}$ is a symmetric matrix which encodes the relative distances in kilometers between the weather stations: $d_{i, j}=(0.1)^{r_{i, j}}$, where $r_{i, j}$ is the distance between the $i$-th and the $j$-th stations, and $r_{i, i}=0$ for every $i$.

We apply the proposed MMDR algorithm for minimizing 30 with $g_{0}=\mu_{0} \mathcal{R}_{1}\left((\cdot)^{-1}\right)$ and $g_{1}=\mu_{1}\|\cdot\|_{1}$. The noise level $\sigma$ is set to the standard deviation of the elements in $\mathbf{W}_{d s}$. Further setting of Algorithm 2 are: $\mu_{0}=1, \mu_{1}=0.1, \gamma_{\ell} \equiv 10, \alpha^{(\ell, k)} \equiv 1$, inner loop tolerance $10^{-5}$ (maximum 3000 iterations) and outer loop 
tolerance $\varepsilon_{o}=10^{-4}$ (maximum 20 iterations). The results are depicted in Fig. 5. Within these settings, the graph is sparse, easy to interpret (cf. Fig. 5). Moreover, a three-subgraphs structure arises: the bigger subgraph is located in the west part, connected to the eastern one by the nodes 19 and 23. In the north a group of 5 stations $(3,5,7,20$ and 28$)$ depicts a subgraph which shares edges with the other twos. Finally, three isolated stations are connected in the north-east part of the map.

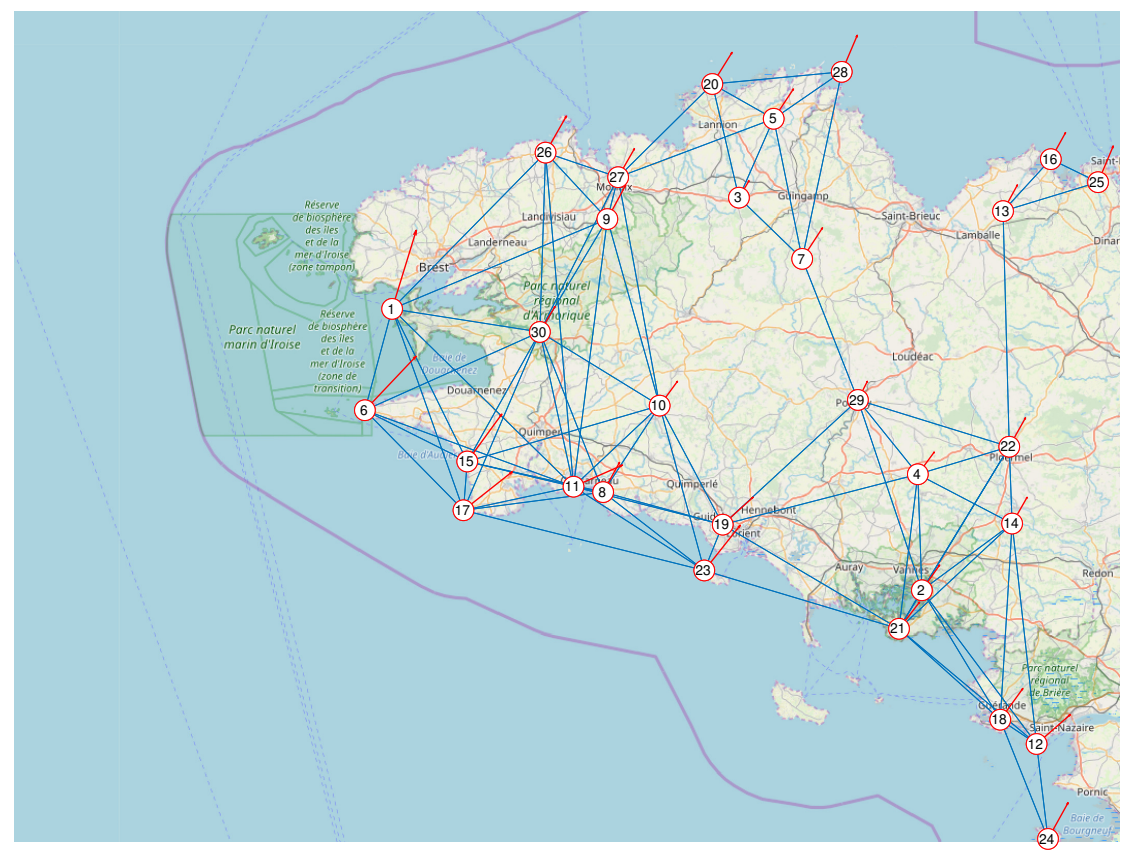

\begin{tabular}{|l|l|}
\hline & Edges \\
& 1 - CAMARET \\
& 2- VANNES-SENE \\
& 3- LOUARGAT \\
& 4- BIGNAN \\
& 5- POMMERIT-JAUDY \\
& 6- PTE DU RAZ \\
& 7- KERPERT \\
& 8- TREGUNC \\
& 9- PLEYBER-CHRIST SA \\
10- GUISCRIFF SA \\
11- BEG_MEIL \\
12- PTE DE CHEMOULIN \\
13- QUINTENIC \\
14- PLEUCADEUC \\
15- PLOVAN \\
16- SAINT-CAST-LE-G \\
17- PTE DE PENMARCH \\
18- GUERANDE \\
19- LORIENT-LANN BIHOUE \\
20- PLOUMANAC'H \\
21- SARZEAU SA \\
22- PLOERMEL \\
23- ILE DE GROIX \\
24- NOIRMOUTIER EN \\
25- DINARD \\
26- SIBIRIL S A \\
$\bigcirc$ 27- MORLAIX_AERO \\
28- ILE-DE-BREHAT \\
29- PONTIVY \\
30- ST-SEGAL S A \\
Wind directionS \\
\hline
\end{tabular}

Figure 5: Recovered graph from wind direction-speed data. Three main subgraphs are present, in the west, in the north and in the south-east. A small group of three stations is connected in the far north. The red arrows represent medium wind direction modulated by the medium speed in the considered time interval.

We now perform comparisons with respect to the classical GLASSO approach, and to the case when $\sigma$ is assumed to be 0. We apply Douglas-Rachford algorithm for minimizing Eq. (1) with $f=-\log \operatorname{det}$, $\left(\forall \mathbf{C} \in \mathcal{S}_{n}^{++}\right) g(\mathbf{C})=\mu_{0} \mathcal{R}_{1}\left(\mathbf{C}^{-1}\right)+\mu_{1}\|\mathbf{C}\|_{1}$. Two settings are considered for the parameters $\left(\mu_{0}, \mu_{1}\right)$, namely $\left(0,5 \times 10^{-6}\right)$ corresponding to GLASSO and $\left(\mu_{0}, \mu_{1}\right)=\left(6 \times 10^{-3}, 10^{-4}\right)$. The algorithm parameters are $\gamma_{\ell} \equiv 4, \alpha^{(\ell, k)} \equiv 1.8$, and the stopping criterion tolerance is $10^{-6}$ with a maximum number of $4 \times 10^{4}$ iterations. The recovered graphs are depicted in Fig. 6. The GLASSO graph seems to provide no useful information, since the degree of the nodes stays rather high. For the second setting of parameters, hereagain, the graph does not show any particular structure. Those comparisons illustrate the advantage of our MMDR method, both accounting for the presence of noise and introducing a spectral penalization. 


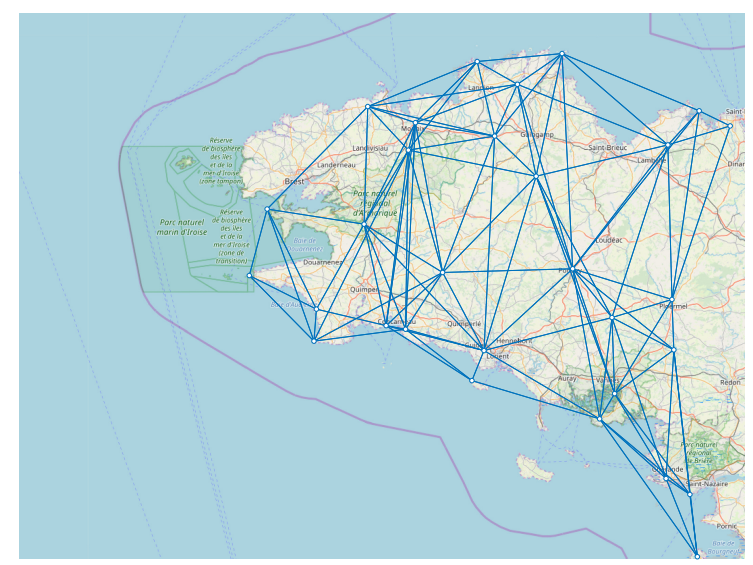

(a) $\left(\mu_{0}, \mu_{1}\right)=\left(0,5 \times 10^{-6}\right)$.

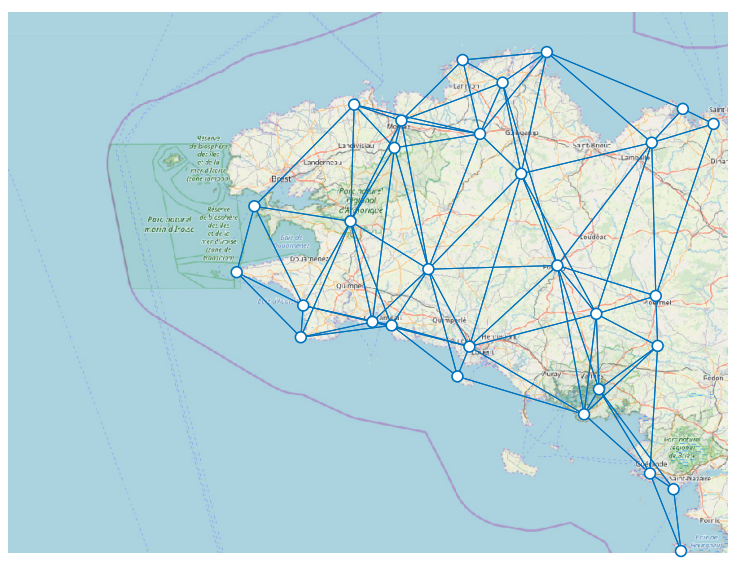

(b) $\left(\mu_{0}, \mu_{1}\right)=\left(6 \times 10^{-3}, 10^{-4}\right)$.

Figure 6: DR algorithm for minimizing (1) with $f=-\log \operatorname{det}$ and $\left(\forall \mathbf{C} \in \mathcal{S}_{n}^{++}\right) g(\mathbf{C})=\mu_{0} \mathcal{R}_{1}\left(\mathbf{C}^{-1}\right)+\mu_{1}\|\mathbf{C}\|_{1}$, for two different settings of regularization parameters.

\section{Conclusions}

In this work, various proximal tools have been introduced to deal with optimization problems involving real symmetric matrices. We have focused on the variational framework (1) which is closely related to the computation of a proximity operator with respect to a Bregman divergence. It has been assumed that $f$ in $(3)$ is a convex spectral function, and $g$ reads as $g_{0}+g_{1}$, where $g_{0}$ is a spectral function. We have provided a fully spectral solution in Section 2 when $g_{1} \equiv 0$, and, in particular, Corollary 1 could be useful for developing algorithms involving proximity operators in other metrics than the Frobenius one. When $g_{1} \not \equiv 0$, a proximal iterative approach has been presented, which is grounded on the use of the Douglas-Rachford procedure. As illustrated by the lists of proximity operators provided for a wide range of choices for $f$ and $g_{0}$, the main advantage of the proposed algorithm is its great flexibility. Numerical experiments show its superiority in terms of convergence speed with respect to two state-of-art algorithms solving the same problem. The proposed matrix estimation framework has also allowed us to introduce a nonconvex formulation of the precision matrix estimation problem arising in the context of noisy graphical lasso. The nonconvexity of the obtained objective function has been circumvented through an MM approach, each step of which consists of solving a convex problem by a Douglas-Rachford sub-iteration. Comparisons with state-of-the-art solutions have demonstrated the robustness of the proposed method. The proposed model and the MM procedure devoted to the minimization of the non-convex functional also reveals to be useful for analyzing real-world multivariate time series from meteorology. It is worth mentioning that all the results presented in this paper could be easily extended to complex Hermitian matrices. It would also be interesting to perform a deeper statistical analysis of the performance of the robust GLASSO approach proposed in this paper. 


\section{Appendix A.}

Proof of Theorem 2, (i) Since it has been assumed that $f$ and $g_{0}$ are spectral functions, we have

$$
\left(\forall \mathbf{C} \in \mathcal{S}_{n}\right) \quad f(\mathbf{C})+g_{0}(\mathbf{C})=\varphi(\mathbf{d})+\psi(\mathbf{d}),
$$

where $\mathbf{d} \in \mathbb{R}^{n}$ is a vector of the eigenvalues of $\mathbf{C}$. It can be noticed that minimizing 12 is obviously equivalent to minimize $\tilde{f}-\gamma^{-1}$ trace $((\overline{\mathbf{C}}+\gamma \mathbf{T}) \cdot)+g_{0}$ where $\widetilde{f}=f+\|\cdot\|_{\mathrm{F}}^{2} /(2 \gamma)$. Then

$$
\widetilde{f}(\mathbf{C})=\widetilde{\varphi}(\mathbf{d}),
$$

where $\widetilde{\varphi}=\varphi+\|\cdot\|^{2} /(2 \gamma)$. Since we have assumed that $\varphi \in \Gamma_{0}\left(\mathbb{R}^{n}\right), \widetilde{\varphi}$ is proper, lower-semicontinuous, and strongly convex. As $\psi$ is lower bounded by an affine function, it follows that

$$
\mathbf{d} \mapsto \widetilde{\varphi}(\mathbf{d})-\gamma^{-1} \boldsymbol{\lambda}^{\top} \mathbf{d}+\psi(\mathbf{d})
$$

is lower bounded by a strongly convex function and it is thus coercive. In addition, $\operatorname{dom} \widetilde{\varphi}=\operatorname{dom} \varphi$, hence $\operatorname{dom} \widetilde{\varphi} \cap \operatorname{dom} \psi \neq \varnothing$. Let us now apply Theorem 1] Let $\widehat{\boldsymbol{\lambda}}$ be a minimizer of $(\mathrm{A} .3)$. It can be claimed that $\widehat{\mathbf{C}}=\mathbf{U} \operatorname{Diag}(\widehat{\boldsymbol{\lambda}}) \mathbf{U}^{\top}$ is a minimizer of $(12)$. On the other hand, minimizing $(A .3)$ is equivalent to minimize $\gamma(\varphi+\psi)+\frac{1}{2}\|\cdot-\boldsymbol{\lambda}\|^{2}$, which shows that $\hat{\boldsymbol{\lambda}} \in \operatorname{Prox}_{\gamma(\varphi+\psi)}(\boldsymbol{\lambda})$.

(ii) If $\psi \in \Gamma_{0}\left(\mathbb{R}^{n}\right)$, then it is lower bounded by an affine function [45, Theorem 9.20]. Furthermore, $\varphi+\psi \in \Gamma_{0}\left(\mathbb{R}^{n}\right)$ and the proximity operator of $\gamma(\varphi+\psi)$ is thus single valued. On the other hand, we also have $\gamma\left(f-\operatorname{trace}(\mathbf{T} \cdot)+g_{0}\right) \in \Gamma_{0}\left(\mathcal{S}_{n}\right)$ [75, Corollary 2.7], and the proximity operator of this function is single valued too. The result directly follows from (i).

\section{Appendix B.}

Proof of Lemma 2. By using differential calculus rules in [76, we will show that the Hessian of $-\mathcal{T}_{\mathbf{S}}$ evaluated at any matrix in $\mathcal{S}_{n}^{++}$is a positive semidefinite operator. In order to lighten our notation, for every invertible matrix $\mathbf{C}$, let us define $\mathbf{M}=\mathbf{C}^{-1}+\sigma^{2} I_{d}$. Then, the first-order differential of $\mathcal{T}_{\mathbf{S}}$ at every $\mathbf{C} \in \mathcal{S}_{n}^{++}$is

$$
\begin{aligned}
\operatorname{dtrace}\left(\mathcal{T}_{\mathbf{S}}(\mathbf{C})\right) & =\operatorname{trace}\left(\left(\mathrm{d} \mathbf{M}^{-1}\right) \mathbf{S}\right) \\
& =\operatorname{trace}\left(-\mathbf{M}^{-1}(\mathrm{~d} \mathbf{M}) \mathbf{M}^{-1} \mathbf{S}\right) \\
& =\operatorname{trace}\left(\left(\mathbf{C}^{-1}+\sigma^{2} \mathrm{I}_{\mathrm{d}}\right)^{-1} \mathbf{S}\left(\mathbf{C}^{-1}+\sigma^{2} \mathrm{I}_{\mathrm{d}}\right)^{-1} \mathbf{C}^{-1}(\mathrm{~d} \mathbf{C}) \mathbf{C}^{-1}\right) \\
& =\operatorname{trace}\left(\left(\mathrm{I}_{\mathrm{d}}+\sigma^{2} \mathbf{C}\right)^{-1} \mathbf{S}\left(\mathrm{I}_{\mathrm{d}}+\sigma^{2} \mathbf{C}\right)^{-1}(\mathrm{~d} \mathbf{C})\right) .
\end{aligned}
$$

We have used the expression of the differential of the inverse [76, Chapter 8, Theorem 3] and the invariance of the trace with respect to cyclic permutations. It follows from (B.1) that the gradient of $\mathcal{T}_{\mathbf{S}}$ reads

$$
\left(\forall \mathbf{C} \in \mathcal{S}_{n}^{++}\right) \quad \nabla \mathcal{T}_{\mathbf{S}}(\mathbf{C})=\left(\mathrm{I}_{\mathrm{d}}+\sigma^{2} \mathbf{C}\right)^{-1} \mathbf{S}\left(\mathrm{I}_{\mathrm{d}}+\sigma^{2} \mathbf{C}\right)^{-1} .
$$


In order to calculate the Hessian $\mathfrak{H}$ of $\mathcal{T}_{\mathbf{S}}$, we calculate the differential of $\nabla \mathcal{T}_{\mathbf{s}}$. Again, in order to simplify our notation, for every matrix $\mathbf{C}$, we define

$$
\mathbf{N}=\mathrm{I}_{\mathrm{d}}+\sigma^{2} \mathbf{C} \quad \Rightarrow \quad \mathrm{d} \mathbf{N}=\sigma^{2} \mathrm{~d} \mathbf{C} .
$$

The differential of $\nabla \mathcal{T}_{\mathbf{S}}$ at every $\mathbf{C} \in \mathcal{S}_{n}^{++}$then reads

$$
\begin{aligned}
\mathrm{d} \operatorname{vect}\left(\nabla \mathcal{T}_{\mathbf{S}}(\mathbf{C})\right)= & \operatorname{vect}\left(\mathrm{d}\left(\mathbf{N}^{-1} \mathbf{S} \mathbf{N}^{-1}\right)\right) \\
= & \operatorname{vect}\left(\left(\mathrm{d} \mathbf{N}^{-1}\right) \mathbf{S} \mathbf{N}^{-1}+\mathbf{N}^{-1}\left(\mathrm{~d} \mathbf{S} \mathbf{N}^{-1}\right)\right) \\
= & -\operatorname{vect}\left(\mathbf{N}^{-1}\left(\mathrm{~d} \mathbf{N}^{-1} \mathbf{N}^{-1} \mathbf{S} \mathbf{N}^{-1}\right)-\operatorname{vect}\left(\mathbf{N}^{-1} \mathbf{S} \mathbf{N}^{-1}(\mathrm{~d} \mathbf{N}) \mathbf{N}^{-1}\right)\right. \\
= & -\left(\left(\mathbf{N}^{-1} \mathbf{S} \mathbf{N}^{-1}\right)^{\top} \otimes \mathbf{N}^{-1}\right) \operatorname{vect}(\mathrm{d} \mathbf{N})+ \\
& -\left(\left(\mathbf{N}^{-1}\right)^{\top} \otimes \mathbf{N}^{-1} \mathbf{S} \mathbf{N}^{-1}\right) \mathrm{d} \operatorname{vect}(\mathbf{N}) \\
= & -\left(\left(\mathbf{N}^{-1} \mathbf{S} \mathbf{N}^{-1}\right) \otimes \mathbf{N}^{-1}+\mathbf{N}^{-1} \otimes\left(\mathbf{N}^{-1} \mathbf{S} \mathbf{N}^{-1}\right)\right) \operatorname{vect}(\mathrm{d} \mathbf{N}) \\
= & \mathfrak{H}(\mathbf{C}) \mathrm{d} \operatorname{vect}(\mathbf{C})
\end{aligned}
$$

with

$$
\mathfrak{H}(\mathbf{C})=-\sigma^{2}\left(\nabla \mathcal{T}_{\mathbf{S}}(\mathbf{C}) \otimes\left(\mathrm{I}_{\mathrm{d}}+\sigma^{2} \mathbf{C}\right)^{-1}+\left(\mathrm{I}_{\mathrm{d}}+\sigma^{2} \mathbf{C}\right)^{-1} \otimes \nabla \mathcal{T}_{\mathbf{S}}(\mathbf{C})\right) .
$$

To derive the above expression, we have used the facts that, for every $\mathbf{A} \in \mathbb{R}^{n \times m}, \mathbf{X} \in \mathbb{R}^{m \times p}$, and $\mathbf{B} \in \mathbb{R}^{p \times q}$, $\operatorname{vect}(\mathbf{A X B})=\left(\mathbf{B}^{\top} \otimes \mathbf{A}\right) \operatorname{vect} \mathbf{X}[76$, Chapter 2,Theorem 2] and that matrices $\mathbf{N}$ and $\mathbf{S}$ are symmetric.

Let us now check that, for every $\mathbf{C} \in \mathcal{S}_{n}^{++}, \mathfrak{H}(\mathbf{C})$ is negative semidefinite. It follows from expression B.2. the symmetry of $\mathbf{C}$, and the positive semidefiniteness of $\mathbf{S}$ that $\nabla \mathcal{T}_{\mathbf{S}}(\mathbf{C})$ belongs to $\mathcal{S}_{n}^{+}$. Since

$$
\begin{aligned}
\left(\nabla \mathcal{T}_{\mathbf{S}}(\mathbf{C}) \otimes\left(\mathrm{I}_{\mathrm{d}}+\sigma^{2} \mathbf{C}\right)^{-1}\right)^{\top} & =\left(\nabla \mathcal{T}_{\mathbf{S}}(\mathbf{C})\right)^{\top} \otimes\left(\left(\mathrm{I}_{\mathrm{d}}+\sigma^{2} \mathbf{C}\right)^{-1}\right)^{\top} \\
& =\nabla \mathcal{T}_{\mathbf{S}}(\mathbf{C}) \otimes\left(\mathrm{I}_{\mathrm{d}}+\sigma^{2} \mathbf{C}\right)^{-1}
\end{aligned}
$$

$\nabla \mathcal{T}_{\mathbf{S}}(\mathbf{C}) \otimes\left(\mathrm{I}_{\mathrm{d}}+\sigma^{2} \mathbf{C}\right)^{-1}$ is symmetric. Let us denote by $\left(\gamma_{i}\right)_{1 \leq i \leq n} \in\left[0,+\infty\left[^{n}\right.\right.$ the eigenvalues of $\nabla \mathcal{T}_{\mathbf{S}}(\mathbf{C})$ and by $\left(\zeta_{i}\right)_{1 \leq i \leq n} \in\left[0,+\infty\left[^{n}\right.\right.$ those of $\mathbf{C}$. According to [76, Chapter 2, Theorem 1], the eigenvalues of $\nabla \mathcal{T}_{\mathbf{S}}(\mathbf{C}) \otimes\left(\mathrm{I}_{\mathrm{d}}+\sigma^{2} \mathbf{C}\right)^{-1}$ are $\left(\gamma_{i} /\left(1+\sigma^{2} \zeta_{j}\right)\right)_{1 \leq i, j \leq n}$ and they are therefore nonnegative. This allows us to claim that $\nabla \mathcal{T}_{\mathbf{S}}(\mathbf{C}) \otimes\left(\mathrm{I}_{\mathrm{d}}+\sigma^{2} \mathbf{C}\right)^{-1}$ belongs to $\mathcal{S}_{n^{2}}^{+}$. For similar reasons, $\left(\mathrm{I}_{\mathrm{d}}+\sigma^{2} \mathbf{C}\right)^{-1} \otimes \nabla \mathcal{T}_{\mathbf{S}}(\mathbf{C}) \in \mathcal{S}_{n^{2}}^{+}$, which allows us to conclude that $-\mathfrak{H}(\mathbf{C}) \in \mathcal{S}_{n^{2}}^{+}$. Hence, we have proved that $\mathcal{T}_{\mathbf{S}}$ is concave on $\mathcal{S}_{n}^{++}$. By continuity of $\mathcal{T}_{\mathbf{S}}$ relative to $\mathcal{S}_{n}^{+}$, the concavity property extends on $\mathcal{S}_{n}^{+}$.

\section{Appendix C.}

Proof of Theorem 5. First note that $\left(\mathbf{C}^{(\ell)}\right)_{\ell \geq 0}$ is properly defined by $(38)$ since, for every $\mathbf{C} \in \mathcal{S}_{n}^{++}$, $\mathcal{G}(\cdot \mid \mathbf{C})$ is a coercive lower-semicontinuous function. It indeed majorizes $\mathcal{F}$ which is coercive, since $f+g_{0}+g_{1}$ has been assumed coercive. 
(i) As a known property of MM strategies, $\left(\mathcal{F}\left(\mathbf{C}^{(\ell)}\right)\right)_{\ell \geq 0}$ is a decaying sequence 69. Under our assumptions, we have already seen that $\mathcal{F}$ has a minimizer. We deduce that $\left(\mathcal{F}\left(\mathbf{C}^{(\ell)}\right)\right)_{\ell \geq 0}$ is lower bounded, hence convergent.

(ii) Since $\left(\mathcal{F}\left(\mathbf{C}^{(\ell)}\right)\right)_{\ell \geq 0}$ is a decaying sequence, $(\forall \ell \geq 0) \mathbf{C}^{(\ell)} \in E$. Since $\mathcal{F}$ is proper, lower-semicontinuous, and coercive, $E$ is a nonempty compact set and $\left(\mathbf{C}^{(\ell)}\right)_{\ell \geq 0}$ admits a cluster point in $E$.

(iii) If $\widehat{\mathbf{C}}$ is a cluster point of $\left(\mathbf{C}^{(\ell)}\right)_{\ell \geq 0}$, then there exists a subsequence $\left(\mathbf{C}^{\left(\ell_{k}\right)}\right)_{k \geq 0}$ converging to $\widehat{\mathbf{C}}$. Since $E$ is a nonempty subset of the relative interior of $\operatorname{dom} g_{0} \cap \operatorname{dom} g_{1}$ and $g_{0}+g_{1} \in \Gamma_{0}\left(\mathcal{S}_{n}\right), g_{0}+g_{1}$ is continuous relative to $E$ [45, Corollary 8.41]. As $f+\mathcal{T}_{\mathbf{S}}$ is continuous on $\operatorname{dom} f \cap \operatorname{dom} \mathcal{T}_{\mathbf{S}}=\mathcal{S}_{n}^{++}, \mathcal{F}$ is continuous relative to $E$. Hence, $\widehat{\mathcal{F}}=\lim _{k \rightarrow+\infty} \mathcal{F}\left(\mathbf{C}^{\left(\ell_{k}\right)}\right)=\mathcal{F}(\widehat{\mathbf{C}})$. On the other hand, by similar arguments applied to sequence $\left(\mathbf{C}^{\left(\ell_{k}+1\right)}\right)_{k \geq 0}$, there exists a subsequence $\left(\mathbf{C}^{\left(\ell_{k_{q}}+1\right)}\right)_{q \geq 0}$ converging to some $\widehat{\mathbf{C}}^{\prime} \in E$ such that $\widehat{\mathcal{F}}=\mathcal{F}\left(\widehat{\mathbf{C}}^{\prime}\right)$. In addition, thanks to $(38)$, we have

$$
\left(\forall \mathbf{C} \in \mathcal{S}_{n}\right)(\forall q \in \mathbb{N}) \quad \mathcal{G}\left(\mathbf{C}^{\left(\ell_{k_{q}}+1\right)} \mid \mathbf{C}^{\left(\ell_{k_{q}}\right)}\right) \leq \mathcal{G}\left(\mathbf{C} \mid \mathbf{C}^{\left(\ell_{k_{q}}\right)}\right) .
$$

By continuity of $f$ and $\nabla \mathcal{T}_{\mathbf{S}}$ on $\mathcal{S}_{n}^{++}$and by continuity of $g_{0}+g_{1}$ relative to $E$,

$$
\left(\forall \mathbf{C} \in \mathcal{S}_{n}\right) \quad \mathcal{G}\left(\widehat{\mathbf{C}}^{\prime} \mid \widehat{\mathbf{C}}\right) \leq \mathcal{G}(\mathbf{C} \mid \widehat{\mathbf{C}}) .
$$

Let us now suppose that $\widehat{\mathbf{C}}$ is not a critical point of $\mathcal{F}$. Since the subdifferential of $\mathcal{G}(\cdot \mid \widehat{\mathbf{C}})$ at $\widehat{\mathbf{C}}$ is $\nabla f(\widehat{\mathbf{C}})+$ $\nabla \mathcal{T}_{\mathbf{S}}(\widehat{\mathbf{C}})+\partial\left(g_{0}+g_{1}\right)(\widehat{\mathbf{C}})[45$, Corollary 16.48(ii)], the null matrix does not belong to this subdifferential, which means that $\widehat{\mathbf{C}}$ is not a minimizer of $\mathcal{G}(\cdot \mid \widehat{\mathbf{C}})$ [5, Theorem 16.3]. It follows from $(\widehat{C} .2$ and standard MM properties that $\mathcal{F}\left(\widehat{\mathbf{C}}^{\prime}\right) \leq \mathcal{G}\left(\widehat{\mathbf{C}}^{\prime} \mid \widehat{\mathbf{C}}\right)<\mathcal{G}(\widehat{\mathbf{C}} \mid \widehat{\mathbf{C}})=\mathcal{F}(\widehat{\mathbf{C}})$. The resulting strict inequality contradicts the already established fact that $\mathcal{F}\left(\widehat{\mathbf{C}}^{\prime}\right)=\mathcal{F}(\widehat{\mathbf{C}})$.

\section{References}

[1] J. C. Duchi, S. Gould, D. Koller, Projected Subgradient Methods for Learning Sparse Gaussians, in: UAI 2008, Proceedings of the 24th Conference in Uncertainty in Artificial Intelligence, Helsinki, Finland, July 9-12, 2008, 2008, pp. 145-152.

[2] S. Ma, L. Xue, H. Zou, Alternating direction methods for latent variable Gaussian graphical model selection, Neural Comput. 25 (8) (2013) 2172-2198. doi:10.1162/NECO_a_00379.

[3] O. Banerjee, L. El Ghaoui, A. d'Aspremont, Model selection through sparse maximum likelihood estimation for multivariate Gaussian or binary data, J. Mach. Learn. Res. 9 (2008) 485-516.

[4] V. Chandrasekaran, P. A. Parrilo, A. S. Willsky, Latent variable graphical model selection via convex optimization, Ann. Statist. 40 (4) (2012) 1935-1967. doi:10.1214/11-A0S949

[5] J. Guo, E. Levina, G. Michailidis, J. Zhu, Joint estimation of multiple graphical models, Biometrika 98 (1) (2011) 1. doi:10.1093/biomet/asq060

[6] J. Friedman, T. Hastie, R. Tibshirani, Sparse inverse covariance estimation with the graphical LASSO, Biostatistics 9 (3) (2008) 432-441. doi:10.1093/biostatistics/kxm045

[7] A. Dempster, Covariance selection, Biometrics 28 (1972) 157-175.

[8] A. d'Aspremont, O. Banerjee, L. E. Ghaoui, First-order methods for sparse covariance selection, SIAM J. Matrix Anal. Appl. 30 (1) (2008) 56-66. doi:10.1137/060670985

[9] J. Bien, R. J. Tibshirani, Sparse estimation of a covariance matrix, Biometrika 98 (4) (2011) 807-820.

[10] Y. Sun, P. Babu, D. P. Palomar, Robust estimation of structured covariance matrix for heavy-tailed elliptical distributions, IEEE Trans. Signal Process. 64 (14) (2016) 3576-3590.

[11] A. Wiesel, Unified framework to regularized covariance estimation in scaled Gaussian models, IEEE Trans. Signal Process. 60 (1) (2012) 29-38. doi:10.1109/TSP.2011.2170685

[12] Y. Sun, P. Babu, D. P. Palomar, Regularized robust estimation of mean and covariance matrix under heavy tails and outliers, in: 2014 IEEE 8th Sensor Array and Multichannel Signal Processing Workshop (SAM), 2014, pp. 125-128. doi:10.1109/SAM.2014.6882356 
[13] N. Meinshausen, P. Bühlmann, High-dimensional graphs and variable selection with the LASSO, Ann. Statist. 34 (3) (2006) 1436-1462. doi:10.1214/009053606000000281

[14] P. Ravikumar, M. J. Wainwright, G. Raskutti, B. Yu, High-dimensional covariance estimation by minimizing $\ell_{1}$-penalized log-determinant divergence, Electron. J. Statist. 5 (2011) 935-980. doi:10.1214/11-EJS631

[15] M. Yuan, Y. Lin, Model selection and estimation in the Gaussian graphical model, Biometrika 94 (1) (2007) 19. doi: 10.1093/biomet/asm018

[16] C. Uhler, Gaussian graphical models: An algebraic and geometric perspective, Tech. rep., https://arxiv.org/abs/1707.04345 (2017).

[17] M. S. Aslan, X.-W. Chen, H. Cheng, Analyzing and learning sparse and scale-free networks using Gaussian graphical models, J. Mach. Learn. Res. 1 (2) (2016) 99-109. doi:10.1007/s41060-016-0009-y

[18] S. Yang, Z. Lu, X. Shen, P. Wonka, J. Ye, Fused multiple graphical LASSO, SIAM J. Optim. 25 (2) (2015) $916-943$. doi:10.1137/130936397.

[19] L. M. Bregman, The Relaxation Method of Finding the Common Point of Convex Sets and Its Application to the Solution of Problems in Convex Programming, USSR Computational Mathematics and Mathematical Physics 7 (1967) $200-217$.

[20] H. H. Bauschke, J. M. Borwein, P. L. Combettes, Bregman monotone optimization algorithms, SIAM J. Control Optim. 42 (2) (2003) 596-636. doi:10.1137/S0363012902407120.

[21] H. H. Bauschke, P. L. Combettes, D. Noll, Joint minimization with alternating Bregman proximity operators, Pac. J. Optim. 2 (3) (2006) 401-424.

[22] C. Brune, A. Sawatzky, M. Burger, Primal and dual Bregman methods with application to optical nanoscopy, Int. J. Comput. Vis. 92 (2) (2011) 211-229. doi:10.1007/s11263-010-0339-5

[23] A. Benfenati, V. Ruggiero, Image regularization for Poisson data, Journal of Physics: Conference Series 657 (1) (2015) 012011 .

[24] A. Benfenati, V. Ruggiero, Inexact Bregman iteration for deconvolution of superimposed extended and point sources, Commun. Nonlinear Sci. Numer. Simul. 20 (3) (2015) 882 - 896. doi:http://dx.doi.org/10.1016/j.cnsns.2014.06.045

[25] X. Zhang, M. Burger, S. Osher, A unified primal-dual algorithm framework based on Bregman iteration, J. Sci. Comput. 46 (1) (2011) 20-46. doi:10.1007/s10915-010-9408-8

[26] W. Yin, S. Osher, D. Goldfarb, J. Darbon, Bregman iterative algorithms for $\ell_{1}$-minimization with applications to compressed sensing, SIAM J. Imaging Sci. 1 (1) (2008) 143-168. doi:10.1137/070703983

[27] T. Goldstein, S. Osher, The split Bregman method for 11-regularized problems, SIAM J. Imaging Sci. 2 (2) (2009) $323-343$. doi: $10.1137 / 080725891$

[28] J. Borwein, A. Lewis, Convex Analysis and Nonlinear Optimization, Springer, 2014.

[29] R. Mazumder, T. Hastie, The graphical LASSO: New insights and alternatives, Electron. J. Stat. 6 (2012) $2125-2149$. doi:10.1214/12-EJS740

[30] A. J. Rothman, P. J. Bickel, E. Levina, J. Zhu, Sparse permutation invariant covariance estimation, Electron. J. Statist. 2 (2008) 494-515. doi:10.1214/08-EJS176.

[31] T. Cai, W. Liu, X. Luo, A constrained $\ell_{1}$ minimization approach to sparse precision matrix estimation, J. Am. Stat. Assoc. 106 (494) (2011) 594-607. doi:10.1198/jasa.2011.tm10155

[32] Y. Nesterov, Smooth minimization of non-smooth functions, Math. Programm. 103 (1) (2005) 127-152. doi:10.1007/ s10107-004-0552-5

[33] K. Scheinberg, S. Ma, D. Goldfarb, Sparse inverse covariance selection via alternating linearization methods, in: Advances in Neural Information Processing Systems 23, 2010, pp. 2101-2109.

[34] C. Wang, D. Sun, K.-C. Toh, Solving log-determinant optimization problems by a Newton-CG primal proximal point algorithm, SIAM J. Optim. 20 (6) (2010) 2994-3013. doi:10.1137/090772514

[35] L. Li, K.-C. Toh, An inexact interior point method for $\ell_{1}$-regularized sparse covariance selection, Math. Program. Comput. 2 (3) (2010) 291-315. doi:10.1007/s12532-010-0020-6

[36] M. Mardani, G. Mateos, G. B. Giannakis, Recovery of low-rank plus compressed sparse matrices with application to unveiling traffic anomalies, IEEE Transactions on Information Theory 59 (8) (2013) 5186-5205. doi:10.1109/TIT.2013.2257913

[37] J. K. Tugnait, Graphical LASSO for high-dimensional complex gaussian graphical model selection, in: ICASSP 2019 2019 IEEE International Conference on Acoustics, Speech and Signal Processing (ICASSP), 2019, pp. 2952-2956. doi: 10.1109/ICASSP.2019.8682867

[38] F. Ong, M. Lustig, Beyond low rank + sparse: Multiscale low rank matrix decomposition, IEEE Journal of Selected Topics in Signal Processing 10 (4) (2016) 672-687.

[39] S.-L. Zhou, N.-H. Xiu, Z.-Y. Luo, L.-C. Kong, Sparse and low-rank covariance matrix estimation, J. Oper. Res. Soc. China 3 (2) (2015) 231-250. doi:10.1007/s40305-014-0058-7

[40] E. Richard, P.-A. Savalle, N. Vayatis, Estimation of simultaneously sparse and low rank matrices, in: Proceedings of the 29th International Conference on Machine Learning (ICML-12), ACM, 2012, pp. 1351-1358.

[41] A. Benfenati, E. Chouzenoux, J.-C. Pesquet, Proximal approaches for matrix optimization problems: application to robust precision matrix estimation. Extended Version., ArXiv e-prints (Jan. 2018). arXiv: 1801.07452

[42] R. Rockafellar, Convex Analysis, Princeton landmarks in mathematics and physics, Princeton University Press, 1970.

[43] J. Moreau, Proximité et dualité dans un espace hilbertien, Bull. Soc. Math. France 93 (1965) $273-299$.

[44] J.-F. Cai, E. J. Candés, Z. Shen, A singular value thresholding algorithm for matrix completion, SIAM J. Optim. 20 (4) (2010) 1956-1982. doi:10.1137/080738970.

[45] H. H. Bauschke, P. L. Combettes, Convex Analysis and Monotone Operator Theory in Hilbert Spaces, 2nd Edition, Springer International Publishing, 2017. doi:10.1007/978-3-319-48311-5

[46] P. L. Combettes, J.-C. Pesquet, Proximal Splitting Methods in Signal Processing, in: Fixed-Point Algorithms for Inverse 
Problems in Science and Engineering, Springer, 2011, pp. 185-212. doi:10.1007/978-1-4419-9569-8

[47] N. Parikh, S. Boyd, Proximal algorithms, Found. Trends Optim. 1 (3) (2014) 127-239. doi:10.1561/2400000003

[48] N. Komodakis, J. C. Pesquet, Playing with duality: An overview of recent primal-dual approaches for solving large-scale optimization problems, IEEE Signal Process. Mag. 32 (6) (2015) 31-54. doi:10.1109/MSP.2014.2377273

[49] M. Burger, A. Sawatzky, G. Steidl, First Order Algorithms in Variational Image Processing, Springer International Publishing, Cham, 2016, pp. 345-407. doi:10.1007/978-3-319-41589-5_10

[50] R. Chartrand, Nonconvex splitting for regularized low-rank + sparse decomposition, IEEE Trans. Signal Process. 60 (2012) $5810-5819$.

[51] C. Chaux, P. L. Combettes, J.-C. Pesquet, V. R. Wajs, A variational formulation for frame-based inverse problems, Inverse Problems 23 (4) (2007) 1495.

[52] E. van den Berg, M. P. Friedlander, Probing the Pareto frontier for basis pursuit solutions, SIAM J. Sci. Comput. 31 (2) (2009) 890-912. doi:10.1137/080714488

[53] L. Condat, Fast projection onto the simplex and the $\ell_{1}$ ball, Math. Programm. 158 (1) (2016) 575-585. doi:10.1007/ s10107-015-0946-6

[54] I. Bengtsson, K. Zyczkowski, Geometry of Quantum States: An Introduction to Quantum Entanglement, Cambridge University Press, Cambridge, 2006. doi:10.1017/CB09780511535048

[55] T. Cover, J. Thomas, Elements of Information Theory, A Wiley-Interscience publication, Wiley, 2006.

[56] R. M. Corless, G. H. Gonnet, D. E. G. Hare, D. J. Jeffrey, D. E. Knuth, On the Lambert W function, Adv. Comput. Math. 5 (1) (1996) 329-359. doi:10.1007/BF02124750

[57] P. L. Lions, B. Mercier, Splitting algorithms for the sum of two nonlinear operators, SIAM J. Numer. Anal. 16 (6) (1979) 964-979. doi:10.1137/0716071

[58] P. L. Combettes, J.-C. Pesquet, A Douglas-Rachford splitting approach to nonsmooth convex variational signal recovery, IEEE J. Sel. Topics Signal Process. 1 (4) (2007) 564-574.

[59] J.-C. Pesquet, N. Pustelnik, A parallel inertial proximal optimization method, Pac. J. Optim. 8 (2) (2012) 273-305.

[60] F. J. Aragón Artacho, J. M. Borwein, Global convergence of a non-convex Douglas-Rachford iteration, J. Global Optim. 57 (3) (2013) 753-769. doi:10.1007/s10898-012-9958-4.

61] G. Li, T. K. Pong, Douglas-Rachford splitting for nonconvex optimization with application to nonconvex feasibility problems, Math. Programm. 159 (1) (2016) 371-401. doi:10.1007/s10107-015-0963-5

[62] C. Chaux, J.-C. Pesquet, N. Pustelnik, Nested iterative algorithms for convex constrained image recovery problem, SIAM J. Imaging Sci. 2 (2) (2009) 730-762.

[63] H. Liu, L. Wang, T. Zhao, Sparse covariance matrix estimation with eigenvalue constraints, J. Comput. Graph. Stat. 23 (2) (2014) 439-459.

[64] Y. Sun, P. Babu, D. P. Palomar, Majorization-Minimization algorithms in signal processing, communications, and machine learning, IEEE Trans. Signal Process. 65 (3) (2017) 794-816. doi:10.1109/TSP.2016.2601299

[65] M. E. Tipping, Sparse Bayesian learning and the relevance vector machine, J. Mach. Learn. Res. 1 (2001) $211-244$. doi: 10.1162/15324430152748236

[66] D. P. Wipf, B. D. Rao, Sparse Bayesian learning for basis selection, IEEE Trans. Signal Process. 52 (8) (2004) $2153-2164$. doi:10.1109/TSP.2004.831016

[67] E. Chouzenoux, J.-C. Pesquet, Convergence Rate Analysis of the Majorize-Minimize Subspace Algorithm, IEEE Signal Process. Lett. 23 (9) (2016) $1284-1288$. doi:10.1109/LSP.2016.2593589

[68] D. R. Hunter, K. Lange, A tutorial on MM algorithms, Amer. Statist. 58 (1) (2004) 30-37. doi:10.1198/0003130042836

[69] M. W. Jacobson, J. A. Fessler, An expanded theoretical treatment of iteration-dependent majorize-minimize algorithms, IEEE Trans. Image Process. 16 (10) (2007) 2411-2422. doi:10.1109/TIP.2007.904387

[70] M. A. T. Figueiredo, J. M. Bioucas-Dias, R. D. Nowak, Majorization-minimization algorithms for wavelet-based image restoration, IEEE Transactions on Image Processing 16 (12) (2007) 2980-2991. doi:10.1109/TIP.2007.909318.

[71] M. Razaviyayn, M. Hong, Z. Luo, A unified convergence analysis of block successive minimization methods for nonsmooth optimization, SIAM Journal on Optimization 23 (2) (2013) 1126-1153. doi:10.1137/120891009

[72] C. F. J. Wu, On the convergence properties of the EM algorithm, Ann. Statist. 11 (1) (1983) 95-103. doi:10.1214/aos/ 1176346060

[73] W. I. Zangwill, Nonlinear programming : a unified approach, Englewood Cliffs, N.J. : Prentice-Hall, 1969.

[74] S. Boyd, N. Parikh, E. Chu, B. Peleato, J. Eckstein, Distributed optimization and statistical learning via the alternating direction method of multipliers, Found. Trends Mach. Learn. 3 (1) (2011) 1-122. doi:10.1561/2200000016

[75] A. S. Lewis, Convex analysis on the Hermitian matrices, SIAM J. Optim. 6 (1) (1996) 164-177. doi:10.1137/0806009

[76] J. R. Magnus, H. Neudecker, Matrix Differential Calculus with Applications in Statistics and Econometrics, 2nd Edition, John Wiley, 1999. 